\title{
Physical conditions in high-redshift GRB-DLA absorbers observed with VLT/UVES: implications for molecular hydrogen searches ${ }^{\star}$
}

\author{
C. Ledoux ${ }^{1}$, P. M. Vreeswijk ${ }^{2}$, A. Smette ${ }^{1}$, A. J. Fox ${ }^{1}$, P. Petitjean ${ }^{3}$, S. L. Ellison ${ }^{4}$, J. P. U. Fynbo ${ }^{2}$, and S. Savaglio ${ }^{5}$ \\ 1 European Southern Observatory, Alonso de Córdova 3107, Casilla 19001, Vitacura, Santiago 19, Chile \\ e-mail: cledoux@eso.org \\ 2 Dark Cosmology Centre, Niels Bohr Institute, University of Copenhagen, Juliane Maries Vej 30, 2100 Copenhagen $\varnothing$, Denmark \\ 3 Institut d'Astrophysique de Paris, CNRS and UPMC Paris 6, UMR 7095, 98 bis boulevard Arago, 75014 Paris, France \\ 4 Department of Physics and Astronomy, University of Victoria, Victoria, BC, V8P 1A1, Canada \\ 5 Max-Planck-Institut für Extraterrestrische Physik, Giessenbachstrasse, PF 1312, 85748 Garching bei München, Germany
}

Received 22 December 2008 / Accepted 15 June 2009

\section{ABSTRACT}

\begin{abstract}
Aims. We aim to understand the nature of the absorbing neutral gas in the galaxies hosting high-redshift long-duration gamma-ray bursts (GRBs) and to determine their physical conditions.

Methods. A detailed analysis of high-quality VLT/UVES spectra of the optical afterglow of GRB 050730 and other Swift-era bursts is presented.

Results. We report the detection of a significant number of previously unidentified allowed transition lines of $\mathrm{Fe}^{+}$, involving the fine structure of the ground term $\left({ }^{6} \mathrm{D}_{7 / 2},{ }^{6} \mathrm{D}_{5 / 2},{ }^{6} \mathrm{D}_{3 / 2},{ }^{6} \mathrm{D}_{1 / 2}\right)$ and that of other excited levels $\left({ }^{4} \mathrm{~F}_{9 / 2},{ }^{4} \mathrm{~F}_{7 / 2},{ }^{4} \mathrm{~F}_{5 / 2},{ }^{4} \mathrm{~F}_{3 / 2},{ }^{4} \mathrm{D}_{7 / 2},{ }^{4} \mathrm{D}_{5 / 2}\right)$, from the $z_{\text {abs }}=3.969, \log N\left(\mathrm{H}^{0}\right)=22.10$, damped Lyman- $\alpha$ (DLA) system located in the host galaxy of GRB 050730. No molecular hydrogen $\left(\mathrm{H}_{2}\right)$ is detected down to a molecular fraction of $\log f<-8.0$. We derive accurate metal abundances for $\mathrm{Fe}^{+}, \mathrm{S}^{+}, \mathrm{N}^{0}$, $\mathrm{Ni}^{+}$, and, for the first time in this system, $\mathrm{Si}^{+}$and $\mathrm{Ar}^{0}$. The absorption lines are best-fit as a single narrow velocity component at $z_{\mathrm{abs}}=3.96857$. The time-dependent evolution of the observed $\mathrm{Fe}^{+}$energy-level populations is modelled by assuming the excitation mechanism is fluorescence following excitation by ultraviolet photons emitted by the afterglow of GRB 050730. This UV pumping model successfully reproduces the observations, yielding a total $\mathrm{Fe}^{+}$column density of $\log N=15.49 \pm 0.03$, a burst/cloud distance (defined to the near-side of the cloud) of $d=440 \pm 30 \mathrm{pc}$, and a linear cloud size of $l=520_{-190}^{+240} \mathrm{pc}$. This application of our photoexcitation code demonstrates that burst/DLA distances can be determined without strong constraints on absorption-line variability provided enough energy levels are detected. From the cloud size, we infer a particle density of $n_{\mathrm{H}} \approx 5-15 \mathrm{~cm}^{-3}$.

Conclusions. We discuss these results in the context of no detections of $\mathrm{H}_{2}$ and $\mathrm{C}$ I lines (with $\left.\log N\left(\mathrm{C}^{0}\right) / N\left(\mathrm{~S}^{+}\right)<-3\right)$ in a sample of seven $z>1.8 \mathrm{GRB}$ host galaxies observed with VLT/UVES. We show that the lack of $\mathrm{H}_{2}$ can be explained by the low metallicities, $[\mathrm{X} / \mathrm{H}]<-1$, low depletion factors, and, at most, moderate particle densities of the systems. This points to a picture where GRB-DLAs typically exhibiting very high $\mathrm{H}^{0}$ column densities are diffuse metal-poor atomic clouds with high kinetic temperatures, $T_{\text {kin }} \gtrsim 1000 \mathrm{~K}$, and large physical extents, $l \gtrsim 100 \mathrm{pc}$. The properties of GRB-DLAs observed at high spectral resolution towards bright GRB afterglows differ markedly from the high metal and dust contents of GRB-DLAs observed at lower resolution. This difference likely results from the effect of a bias, against systems of high metallicity and/or close to the GRB, due to dust obscuration in the magnitudelimited GRB afterglow samples observed with high-resolution spectrographs.
\end{abstract}

Key words. gamma rays: bursts - Galaxy: abundances - galaxies: ISM - galaxies: quasars: absorption lines cosmology: observations

\section{Introduction}

Thanks to prompt response, most notably on $8-10 \mathrm{~m}$-class ground-based telescopes, high-resolution optical spectroscopy of rapidly fading long-duration ( $\gtrsim 2 s$ ) gamma-ray burst (GRB) afterglows provides detailed information on the kinematics, chemical abundances, and physical state of the gaseous component of circumburst, host galaxy interstellar and intergalactic matter intervening these lines of sight (e.g., Jakobsson et al. 2004). At the GRB host-galaxy redshift, large amounts of

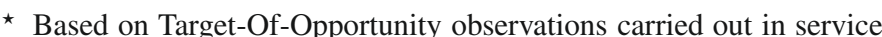
mode under progs. ID 075.A-0603, P.I. Fiore, and 075.A-0385, 077.D0661, 080.D-0526, and 081.A-0856, P.I. Vreeswijk, with the Ultraviolet and Visual Echelle Spectrograph (UVES) installed at the Nasmyth-B focus of the Very Large Telescope (VLT), Unit 2 - Kueyen, operated by the European Southern Observatory (ESO) on Cerro Paranal in Chile.
}

neutral, singly, and highly ionized gas are usually seen (e.g., Fiore et al. 2005; Prochaska et al. 2007b; Fox et al. 2008), including the highest neutral hydrogen column densities measured to date at high redshift from UV absorption-line spectroscopy (e.g., Vreeswijk et al. 2004; Chen et al. 2005; Berger et al. 2006). While such high $\mathrm{H}^{0}$ column densities suggest an origin in the immediate surroundings of the GRB and/or the inner regions of its host, the overall $\mathrm{H}^{0}$ column density distribution of the absorbers is fairly flat (Jakobsson et al. 2006), with values as low as $\approx 10^{17}$ up to $10^{22.6}$ atoms $\mathrm{cm}^{-2}$ (Prochaska et al. 2008; Watson et al. 2006), illustrating the diversity of environments probed by the observations. Metallicities measured in the $\mathrm{H}^{0}$ gas vary widely from less than $1 / 100$ th of solar to nearly solar - with a tendency for low metallicities - (Fynbo et al. 2006; Prochaska et al. 2007b), consistent with an origin in dwarf and/or low-mass galaxies and the currently favoured scenario for long-duration 
GRBs, the collapse of a massive Wolf-Rayet star endowed with rotation (Woosley 1993; MacFadyen \& Woosley 1999; Woosley \& Heger 2004).

Several nearby long-duration GRBs have now been shown to be associated with supernovae explosions (Galama et al. 1998; Stanek et al. 2003; Hjorth et al. 2003; Malesani et al. 2004; Pian et al. 2006), providing the most direct evidence that the progenitors of long-duration GRBs are indeed massive stars. As most star formation occurs within molecular clouds, it is generally expected that the latter are the birthplaces of many GRBs. However, although various studies (Galama \& Wijers 2001; Reichart \& Price 2002) have argued in favour of a link between molecular clouds and GRBs, compelling observational evidence such as the detection of molecular hydrogen $\left(\mathrm{H}_{2}\right)$ in GRB optical spectra is lacking (Vreeswijk et al. 2004; Tumlinson et al. 2007). As absorption lines related to ground-state or vibrationally excited $\mathrm{H}_{2}$ energy levels should be detectable (Draine \& Hao 2002), the absence of detection so far suggests that any $\mathrm{H}_{2}$ molecules in the immediate vicinity of a GRB are dissociated by the intense X-ray/UV afterglow flux. However, an H II region around the progenitor, or its star cluster, would also be sufficient to ionize $\mathrm{H}^{0}$ and dissociate $\mathrm{H}_{2}$ up to distances of $50-100 \mathrm{pc}$ (Whalen et al. 2008).

Unlike intervening QSO absorbers and damped Lyman- $\alpha$ (DLA) systems $\left(N\left(\mathrm{H}^{0}\right) \geq 10^{20.3}\right.$ atoms $\left.\mathrm{cm}^{-2}\right)$ observed in QSO spectra, strong absorption lines involving fine-structure and other metastable levels of ions such as $\mathrm{O}^{0}, \mathrm{Si}^{+}$, and $\mathrm{Fe}^{+}$ are ubiquitous in GRB-DLAs (Vreeswijk et al. 2004; Chen et al. 2005; Berger et al. 2006; Penprase et al. 2006; Prochaska et al. 2006, 2007a; D'Elia et al. 2007; Vreeswijk et al. 2007; Thöne et al. 2008). These levels could be populated by collisions with electrons if the particle density per unit volume is high enough, by indirect excitation by IR photons, or by the UV flux from the GRB afterglow. In Vreeswijk et al. (2007), the detection of the time variability of absorption lines (see also Dessauges-Zavadsky et al. 2006) involving the fine structure of the ground term and other metastable energy levels of both $\mathrm{Fe}^{+}$ and $\mathrm{Ni}^{+}$towards GRB 060418 was successfully modelled. This demonstrated that UV pumping followed by de-excitation cascades is the mechanism at play to form these lines as suggested by Prochaska et al. (2006). The burst/DLA distance inferred in the above case, $d=1.7 \mathrm{kpc}$, represented the first determination of the distance of the bulk of the absorbing neutral material to a GRB explosion site. This is consistent with lower limits on the distance, $d \gtrsim 50 \mathrm{pc}$, derived from the observation of strong associated Mg I absorption in, e.g., GRB 051111, which excludes significant photo-ionization effects (Prochaska et al. 2006).

Searches for molecular hydrogen in GRB-DLAs, thus equivalently in the interstellar medium (ISM) of GRB host galaxies, have led to negative results till now, though in two cases, towards GRB 050401 and GRB 060206, tentative evidence of $\mathrm{H}_{2}$ lines has been reported (Watson et al. 2006; Fynbo et al. 2006). The $\mathrm{H}_{2}$ column-density upper limits derived in a handful of cases from the non-detection of UV absorption lines are somewhat surprising given the very high $\mathrm{H}^{0}$ column densities observed in some GRB-DLAs. Indeed, in the Galactic ISM such clouds are found to be nearly fully molecular (see, e.g., Savage et al. 1977; Jenkins \& Shaya 1979). However, several factors could reduce the amount of $\mathrm{H}_{2}$ present in DLAs: low metallicity, low particle density, strong ambient UV radiation field, and/or, in the case of GRBs, the UV flux from the afterglow itself. Tumlinson et al. (2007) argue that the influence of the latter is negligible and that a combination of low metallicity and extreme UV radiation field from nearby star-forming regions - expected to be present in
GRB hosts - must be invoked to explain not detecting $\mathrm{H}_{2}$ along GRB lines of sight (see also Whalen et al. 2008). However, given the extremely small GRB-DLA sample size and the handful of systems considered so far, it is still unclear whether $\mathrm{H}_{2}$ in GRB host galaxies is really deficient compared to QSO-DLAs and, if so, why.

In this paper, we present an independent, thorough analysis of high-quality UVES spectra of GRB 050730 secured by Fiore et al. (ESO prog. ID 075.A-0603) at two consecutive epochs after the burst. This line of sight exhibits one of the highest $\mathrm{H}^{0}$ column density DLAs ever observed in GRB afterglow spectra (Starling et al. 2005; Chen et al. 2005; D'Elia et al. 2007) with a metallicity at the low end of the distribution for GRB hosts (see Chen et al. 2005; Prochaska et al. 2007a; D'Elia et al. 2007; see also this paper, Sect. 2.2.1). For the first time, we identify in the spectrum of this GRB afterglow absorption lines that originate from numerous $\mathrm{Fe}^{+}$metastable energy levels. In addition, we self-consistently model the time-dependent evolution of the energy-level populations. This allows us to determine the distance of the bulk of the neutral gas to the GRB explosion site and the size of the absorbing cloud, and to address the question of whether the GRB 050730 afterglow flux is responsible for the observed stringent upper limit on the $\mathrm{H}_{2}$ molecular fraction. We then discuss in a more general context the non-detections of $\mathrm{H}_{2}$ molecules taking advantage of the current sample of seven VLT/UVES spectra of Swift-era, $z>1.8$, GRB host absorbers. We compare them to QSO-DLAs where $\mathrm{H}_{2}$ has been searched for at high spectral resolution to gain insights into the physical conditions prevailing in GRB-DLAs.

This paper is organised as follows. In Sect. 2.1, we recall basic facts on the observations and explain the data reduction process. In Sect. 2.2, we present a detailed analysis of absorption lines from the GRB host galaxy to constrain the metallicities, $\mathrm{H}_{2}$ content, and photo-excitation of $\mathrm{Fe}^{+}$in the absorbing cloud. We model the time-dependent evolution of the $\mathrm{Fe}^{+}$metastable energy-level populations in Sect. 2.3. In Sect. 3, we use $\mathrm{H}^{0}$ photo-ionization and $\mathrm{H}_{2}$ photo-dissociation calculations to assess the influence of the GRB afterglow radiation and the non-detections of $\mathrm{H}_{2}$ in GRB-DLAs in the most up-to-date sample of VLT/UVES spectra. This enables us to discuss the physical nature of GRB-DLAs. Finally, we summarise our findings and comment on future prospects in Sect. 4.

\section{GRB 050730}

\subsection{Observations and data reduction}

GRB 050730 was observed with the Ultraviolet and Visual Echelle Spectrograph (UVES, Dekker et al. 2000) mounted at the Nasmyth-B focus of the ESO VLT, UT 2 - Kueyen, $8.2 \mathrm{~m}$ telescope on Cerro Paranal observatory. The data were gathered in service mode through a classical Target-of-Opportunity request by the programme of Fiore et al. (ESO prog. ID 075.A0603). Two $3000 \mathrm{~s}$ exposures were taken consecutively as soon as possible after evening twilight on July 31, 2005, at 00:32 UT and 1:27 UT (mid-exposure times), corresponding to, respectively, 4.57 and $5.48 \mathrm{~h}$ after the burst. The approximate afterglow I-band magnitude at the mid-exposure time of these two epochs is $I=17.6$ and $I=18.0$ (Pandey et al. 2006). Standard instrument configurations with, respectively, Dichroic \#1 and Dichroic \#2 were used (both of them using the Blue and Red spectroscopic arms simultaneously; see also D'Elia et al. 2007). The first-epoch observations cover the wavelength ranges of 3050-3850 and 4800-6800 $\AA$ and the second epoch covers the 
$3850-4800$ and $6800-10000 \AA$ intervals. There are small overlaps between the two-epoch spectra and, in addition, two $\sim 100 \AA$ gaps in the Red due to the physical separation between the two Red CCD detectors. CCD pixels were binned $2 \times 2$, and the spectrograph entrance slit width was fixed to $1^{\prime \prime}$ to match the ambient seeing conditions leading to a resolving power of $\approx 7 \mathrm{~km} \mathrm{~s}^{-1}$ $(F W H M)$.

The data were reduced using standard techniques for echelle data processing with the public version, v2.2.0, of the UVES pipeline ${ }^{1}$ based on the ESO MIDAS data reduction package. The main characteristics of the UVES pipeline are to perform robust inter-order background subtractions for master flat-fields and science frames, and to allow for optimal extraction of the object signal performing sky subtraction and rejecting cosmic rays simultaneously. The pipeline products were checked step by step. The wavelength scale of the reduced spectra was then converted to vacuum-heliocentric values. Due to the variable nature of the source, individual exposures were not co-added. The typical signal-to-noise ratio per pixel achieved in each of the two reduced afterglow spectra lies in the range $8-15$ over the 4600-9000 Å interval.

\subsection{Data analysis}

The GRB 050730 line of sight is characterized by the presence of an extremely strong DLA absorber at $z=3.969$. As no higher redshift system is observed and $\mathrm{Fe}$ II lines from metastable levels are also detected, we assume this is the GRB host-galaxy redshift. The total neutral hydrogen column density of the GRB 050730-DLA has been measured in previous analyses (Starling et al. 2005; Chen et al. 2005; D'Elia et al. 2007) and the standard deviation of the three available measurements, based on different datasets, is very small $(0.04 \mathrm{dex})$. We thus adopt their average value and combined uncertainty, i.e., $\log N\left(\mathrm{H}^{0}\right)=22.10 \pm 0.10$. This is fully consistent with our own measurement based on the UVES data. Numerous metal absorption lines from neutral, singly, and highly ionized species associated to the GRB-DLA are detected in the UVES spectra. While D'Elia et al. (2007) have studied the multi-component structure of the absorption profiles from a number of transition lines using the same dataset, we will restrict our analysis to weak or at most mildly saturated lines. For instance, C II $\lambda 1334, \mathrm{C}$ II $^{\star} \lambda 1335$, O I $\lambda 1302, \mathrm{O}^{\star} \lambda 1304, \mathrm{O}^{\star \star} \lambda 1306, \mathrm{Si}$ II $\lambda 1304$, and Si II $\lambda 1309$ ground-term fine-structure lines are detected but they are heavily saturated and, therefore, cannot be used to derive accurate column density or velocity information.

The overall metal absorption profiles from neutral and singly ionized species at the GRB host-galaxy redshift are dominated by a single narrow velocity component at $z_{\text {abs }}=3.96857$. Interestingly, this component alone represents $\gtrsim 90 \%$ of the total optical depth of non-saturated lines. This makes the Voigtprofile modelling of non-saturated lines from the GRB 050730DLA cloud, as shown below, exceptionally simple and robust.

\subsubsection{Metallicities}

We first focus on neutral and singly ionized species excluding $\mathrm{Fe}^{+}$, which we discuss in Sect. 2.2.3. Among previous analyses (Chen et al. 2005; Prochaska et al. 2007a; D'Elia et al. 2007), column density measurements from unsaturated lines were derived by Prochaska et al. (2007a) for $\mathrm{S}^{+}, \mathrm{N}^{0}$, and $\mathrm{Ni}^{+}$. In this

\footnotetext{
${ }^{1}$ http://www.eso.org/projects/dfs/dfs-shared/web/vlt/ vlt-instrument-pipelines.html
}

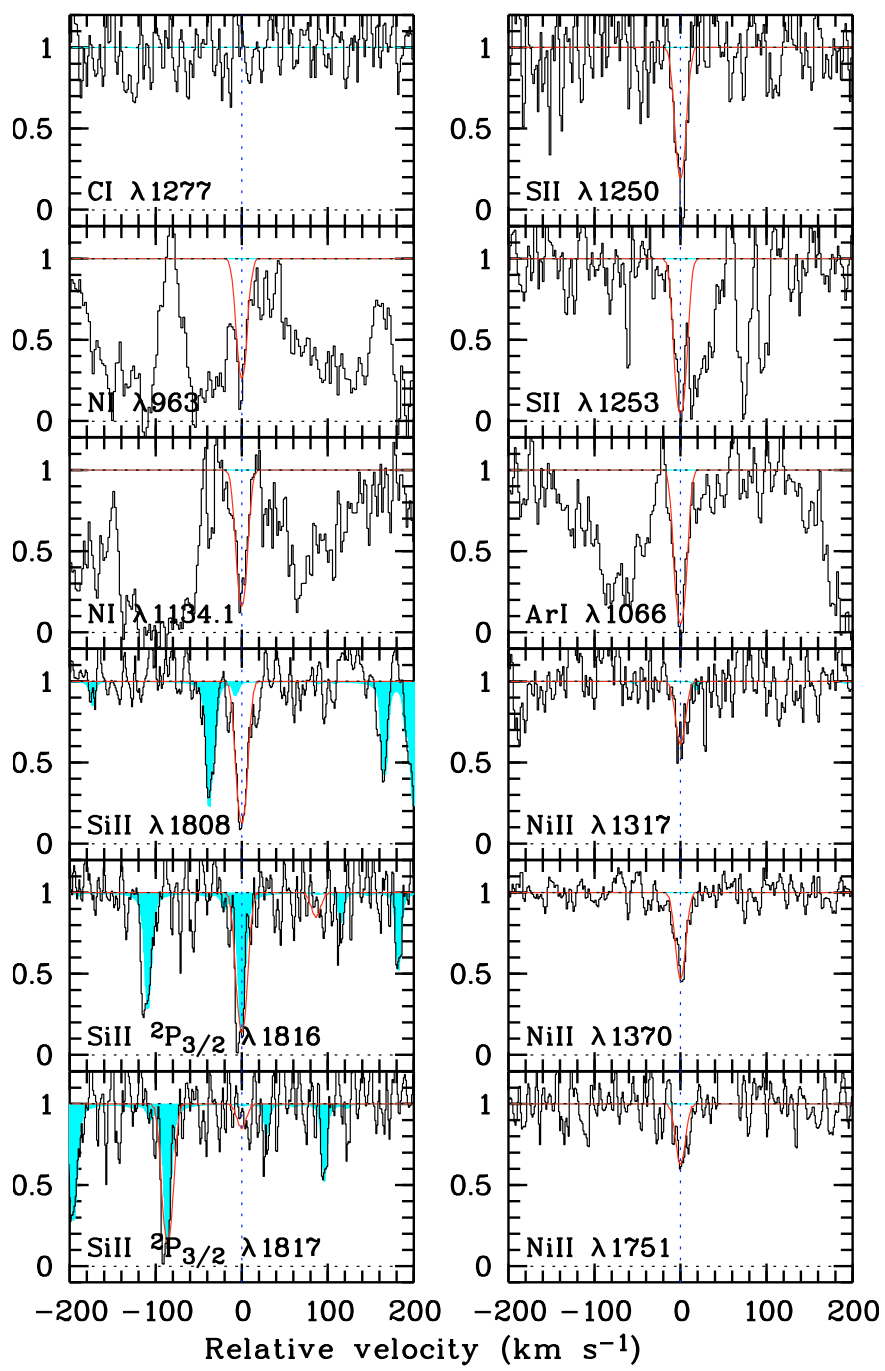

Fig. 1. Normalized UVES spectral portions around weaker lines from neutral and singly ionized species at the GRB 050730-DLA redshift of $z_{\mathrm{abs}}=3.96857$ (taken as the origin of the velocity scale). Transition lines with rest-frame wavelengths shorter than $1350 \AA$ were observed at the first epoch and the other lines at the second epoch. The best Voigt-profile fit is overplotted. The column density from Ni II $\lambda 1317$, detected in the first-epoch data, has been determined independently of the other two Ni II lines observed at the second epoch. The same turbulentbroadening parametre value has been used for all lines and epochs. The shaded regions in the panels showing the Si II lines indicate the expected location and shape of atmospheric features from a synthetic telluric absorption-line spectrum described in Sect. 2.2.3.

work, we perform simultaneous Voigt-profile fits to the Si II, Si II ${ }^{\star}, \mathrm{S}$ II, N I, Ar I, and Ni II lines detected in the UVES spectrum and, with the exception of $\mathrm{Si}^{\star}$ II $^{\star}$ (see below), weak and unblended enough to allow for an accurate determination of column densities. The GRB afterglow continuum was normalized locally around the transition lines of interest using spectral regions with a typical width of $1000 \mathrm{~km} \mathrm{~s}^{-1}$. In order to fit the lines, the redshift and the (assumed-to-be) purely turbulentbroadening parametre values were required to be the same for all lines and the two epochs of observations. The atomic data compiled by Morton (2003) were used for all studied transitions, except for the oscillator strengths of Ni II $\lambda \lambda 1317,1370$ (Jenkins \& Tripp 2006) and the rest-frame wavelengths of S II $\lambda \lambda 1250,1253$ (Morton 1991). The spectra and best Voigt-profile fits are shown in Fig. 1 and the results summarised in Table 1. The best-fit 
Table 1. Ionic column densities of neutral and singly ionized species at the GRB 050730-DLA redshift of $z_{\mathrm{abs}}=3.96857$.

\begin{tabular}{lllc}
\hline \hline Ion & Energy level & $\begin{array}{l}\text { Transition } \\
\text { lines used }(\AA)\end{array}$ & $\log N \pm \sigma_{\log N}{ }^{b}$ \\
\hline \multicolumn{4}{l}{ 1st epoch of observations: } \\
$\mathrm{C}^{0}$ & g.s. & \\
$\mathrm{N}^{0}$ & g.s. & 1277 & $<13.05^{c}$ \\
$\mathrm{~S}^{+}$ & g.s. & $963,1134.1$ & $14.77 \pm 0.03$ \\
$\mathrm{Ar}^{0}$ & g.s. $^{a}$ & 1250,1253 & $15.11 \pm 0.04$ \\
$\mathrm{Ni}^{+}$ & g.s. & 1066 & $14.36 \pm 0.05$ \\
\hline $2 \mathrm{nd}$ & epoch of observations: & $13.53 \pm 0.05$ \\
$\mathrm{Si}^{+}$ & g.s. $^{a}\left({ }^{a} \mathrm{P}_{1 / 2}\right)$ & 1808 & \\
$\mathrm{Si}^{+}$ & ${ }^{2} \mathrm{P}_{3 / 2}$ & 1816,1817 & $15.47 \pm 0.03$ \\
$\mathrm{Ni}^{+}$ & g.s. & $<15.55^{d}$ \\
\hline
\end{tabular}

${ }^{a}$ Ground state. ${ }^{b}$ Listed uncertainties are the formal errors provided by FITLYMAN. ${ }^{c} 3 \sigma$ upper limit. ${ }^{d}$ Blend with telluric absorption.

turbulent-broadening parametre value is $b=6.5 \pm 0.2 \mathrm{~km} \mathrm{~s}^{-1}$. The column density measurement uncertainties given in the table are the formal errors provided by FITLYMAN. They do not include possible additional uncertainties related to the continuum placement. Throughout the paper, we adopt the solar system abundances recommended in Lodders (2003).

Chen et al. (2005) estimated the overall metallicity of the GRB 050730-DLA from the column density of sulphur, $\log N\left(\mathrm{~S}^{+}\right)=15.34 \pm 0.09$, further refined to be $15.22 \pm 0.06$ by Prochaska et al. (2007a). Although not considered in the analysis of D'Elia et al. (2007), S II lines are also detected in the UVES dataset. In the latter, the $1250 \AA$ feature reaches zero residual intensity (see Fig. 1), which is not the case in the MIKE spectra acquired practically simultaneously. While the UVES spectrum is affected by a narrow spike at the location of the S II $\lambda 1250$ line, it is generally of significantly higher quality in terms of $\mathrm{S} / \mathrm{N}$ ratio and spectral resolution as can be seen from the comparison of Fig. 1 with Figs. 2 and 3 from Prochaska et al. (2007a; or equivalently Fig. 2 from Chen et al. 2005). A simultaneous fit of the S II $\lambda \lambda 1250,1253$ lines in the first-epoch UVES spectrum leads us to derive a column density of $15.11 \pm 0.04$, and a corresponding metallicity of $[\mathrm{S} / \mathrm{H}]=-2.18 \pm 0.11$, somewhat lower than the previous estimates. The higher estimate by Prochaska et al. (2007a) is probably caused by the use of the apparent optical depth method on the blended S II $\lambda 1253$ line (see Fig. 1).

Our result for sulphur is consistent with the $\mathrm{Si}^{+}$metallicity we derive from the second-epoch UVES observations: $[\mathrm{Si} / \mathrm{H}]=$ $-2.17 \pm 0.10$. However, we note that there could be some contribution to the total $\mathrm{Si}^{+}$column density from the ${ }^{2} \mathrm{P}_{3 / 2}$ finestructure level of the ground term. The expected position of the Si II $\lambda 1816$ line is badly blended with a telluric absorption feature (see Fig. 1; see also Sect. 2.2.3 for details on the telluric-line spectrum). Therefore, we shall consider in Table 1 the result of Voigt-profile fitting of this line strictly as an upper limit. Given the strength of the blending feature the actual $\mathrm{Si}^{+}$fine-structure level column density could be at least an order of magnitude smaller than the value reported in Table 1 . The $3 \sigma$ columndensity upper limit derived from the non-detection of the much weaker $\lambda 1817$ line is 0.15 dex higher than this value $(<15.7)$.

Singly ionized nickel is detected in the UVES spectra at the two epochs of observations. $\mathrm{Ni}^{+}$column densities were derived independently, from the $1317 \AA$ line detected in the first-epoch spectrum, and from the other two Ni II lines $(\lambda \lambda 1370,1751)$ observed at the second epoch (see Table 1$)$. The resulting values differ by 0.16 dex $(3 \sigma)$ in the sense of column densities

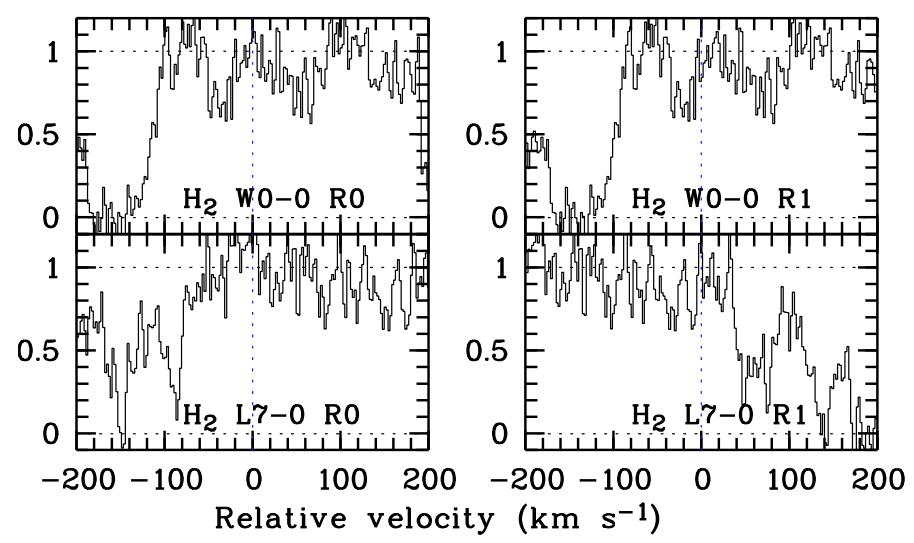

Fig. 2. Normalized UVES spectra around several strong transitions to the $J=0$ and 1 rotational levels of the vibrational ground-state of $\mathrm{H}_{2}$ molecules at the GRB 050730-DLA redshift of $z_{\mathrm{abs}}=3.96857$ (taken as the origin of the velocity scale) in the first-epoch observations.

becoming higher with time. However, given that the uncertainties listed in Table 1 do not include errors on the continuum placement, this result has lower significance. The column density derived from the Ni II line equivalent widths measured by Prochaska et al. (2007a), using the sum of their three spectra taken on average $4.82 \mathrm{~h}$ (mid-exposure time) after the burst, is $\log N\left(\mathrm{Ni}^{+}\right)=13.56 \pm 0.06$. This is consistent with our measurements. Finally, we note that, due to the lack of spectral coverage of the corresponding transition lines, the metastable-level populations of singly ionized nickel (see Vreeswijk et al. 2007) cannot be constrained.

Transition lines from neutral nitrogen and neutral argon are detected in the first-epoch UVES spectrum in a crowded Lyman- $\alpha$ forest but the lines are narrow and well-defined. We are therefore confident that the measured column densities (see Table 1) are reliable. The $\mathrm{N}^{0}$ column density also agrees with the one derived by Prochaska et al. (2007a) in the averaged MIKE spectrum. Finally, the strong C I $\lambda 1277$ transition line is undetected in the UVES spectrum down to a $3 \sigma$ column-density upper limit of 13.05 (see Fig. 1).

\subsection{2. $\mathrm{H}_{2}$ content}

From the high quality of the UVES data, we can set a stringent upper limit on the presence of molecular hydrogen in the GRB 050730-DLA. $\mathrm{H}_{2}$ in its vibrational ground-state is undetected at $3 \sigma$ confidence down to column densities of $\log N\left(\mathrm{H}_{2}\right)=$ 13.30 and 13.65 for, respectively, the $J=0$ and 1 rotational levels in the first-epoch spectrum taken $4.6 \mathrm{~h}$ (mid-exposure time) after the burst (see Fig. 2). The expected positions of the Lyman- and Werner-bands of $\mathrm{H}_{2}$ are not covered by the secondepoch observations. We used the oscillator strengths from the Meudon group ${ }^{2}$ based on calculations described in Abgrall et al. (1994). These upper limits translate to a molecular fraction, $f \equiv 2 N\left(\mathrm{H}_{2}\right) /\left(2 N\left(\mathrm{H}_{2}\right)+N\left(\mathrm{H}^{0}\right)\right)<10^{-8.0}$, for the sum of the first two rotational levels. This is a factor of ten deeper than the constraint previously derived by Tumlinson et al. (2007) from the aforementioned MIKE data. This is also the tightest constraint ever obtained for any extragalactic line of sight at high redshift including the database of 77 QSO-DLAs from Noterdaeme et al. (2008). We do not find evidence in the UVES spectra of vibrationally excited $\mathrm{H}_{2}$ lines as would be expected for dense cold

2 http://amrel.obspm.fr/molat/ 
molecular clouds illuminated by incident GRB afterglow radiation (Draine \& Hao 2002). This is consistent with the extremely low molecular fraction derived above from the non-detection of transition lines to the $\mathrm{H}_{2}$ vibrational ground-state.

\subsection{3. $\mathrm{Fe}^{+}$ground-state and metastable levels}

In addition to the study of metal absorption lines commonly observed in QSO-DLAs (see Sect. 2.2.1), we have systematically searched for all absorption features detected at $3 \sigma$ confidence in the UVES spectra and cross-checked their possible identification using the line lists from Morton (2003). To avoid false identifications due to sky absorption lines, we employed synthetic telluric absorption-line spectra. The latter were obtained with an IDL routine based on the Reference Forward Model (RFM) ${ }^{3}$, a line-by-line radiative transfer code, using the 2004 edition, v12.0, of the high-resolution transmission molecular absorption (HITRAN) database (Rothman et al. 2005). Featured components of RFM include an atmospheric profile describing mean pressure, temperature, and molecular concentrations for typically 50 atmospheric layers. Following Smette et al. (2008), we have calculated synthetic telluric absorption-line spectra for an amount of precipitable water vapour of $2.0 \mathrm{~mm}$ (resp. $2.4 \mathrm{~mm}$ ) and a mean airmass of 1.3 (resp. 1.6) corresponding to the conditions prevailing during the first-epoch (resp. second-epoch) UVES observations of GRB 050730.

In the line identification process, we uncovered the origin of 18 previously unidentified narrow absorption-line features which are transition lines from the fine-structure levels of the ground term and other metastable energy levels of $\mathrm{Fe}^{+}$at the GRB host-galaxy redshift of $z_{\mathrm{abs}}=3.96857$. This is confirmed by the detection of typically two or more well-defined lines with consistent optical depths, and a cross-correlation of their measured rest-frame wavelengths with the NIST atomic spectra database ${ }^{4}$.

In order to fit the lines, we first normalized the spectra around each line locally in an objective manner using a customized routine which calculates the median value of the afterglow continuum after rejection of significant $(\geq 3 \sigma)$ absorption and emission features. Note that, in spectral regions heavily affected by telluric absorption, most notably at rest-frame wavelengths of around $1640 \AA$ (see below), this procedure tends to underestimate the level of the continuum. We assumed that the broadening parametre was purely turbulent. Its value as well as the redshift were assumed to be identical for all lines and the two epochs of observations. However, the column densities were allowed to be different for different energy levels and, for a given energy level, different from one epoch to the other. The possibility that $\mathrm{Fe}^{+}$column densities might vary with time has not been considered in previous analyses of GRB 050730. For consistency with the time-dependent photo-excitation modelling of the observed $\mathrm{Fe}^{+}$level populations presented below, which demands the inclusion of as many energy levels as possible (see details in Sect. 2.3), we have considered the more complete set of atomic parametre values from the Cloudy input file based on the Opacity Project (Verner \& Ferland 1996; Verner et al. 1999). In view of this modelling, care has been exercised to derive column density measurement errors taking into account the uncertainties in the continuum placement. These uncertainties indeed dominate the formal errors provided by FITLYMAN in the case of weak lines. As a consequence of this, and contrary to Sect. 2.2.1,

\footnotetext{
3 http://wwW.atm.ox.ac.uk/RFM/

${ }^{4}$ http://physics.nist.gov/PhysRefData/ASD/index.html
}

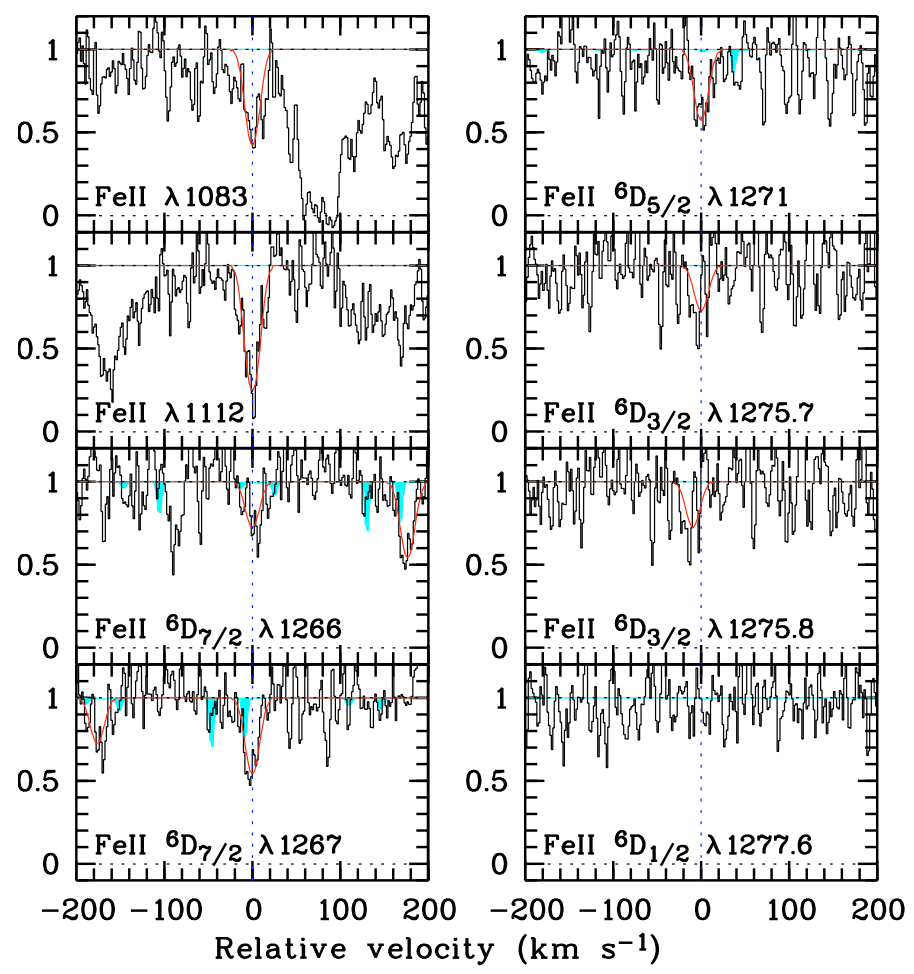

Fig. 3. Voigt-profile fitting to transition lines from the fine-structure levels of the $\mathrm{Fe}^{+}$ground-term at the GRB 050730-DLA redshift of $z_{\text {abs }}=3.96857$ (taken as the origin of the velocity scale) in the firstepoch UVES observations. The lower level of the transitions, for which the column density is determined from the fits, is indicated in each panel, except for the lowest $\mathrm{Fe}^{+}$energy level (ground state) corresponding to ${ }^{6} \mathrm{D}_{9 / 2}$. The shaded regions in some of the panels indicate the expected location and shape of atmospheric features from a synthetic telluric absorption-line spectrum described in Sect. 2.2.3.

errors have been estimated from the line fitting using the best-fit continuum normalisation (see above) comparing the results with Voigt-profile fits with the continuum placed at reasonable upper and lower boundaries. We found these to correspond to adjusting the continuum level by plus or minus 0.5 times the noise rms in the continuum adjacent to the line. The results of Voigt-profile fitting together with the list of lines used for the fits are shown in Figs. 3 and 4, and summarised in Table 2. The best-fit turbulentbroadening parametre value is $b=9.9 \pm 1.1 \mathrm{~km} \mathrm{~s}^{-1}$. Note that the Fe II lines were fitted separately from the other metal lines resulting in a somewhat different $b$ value (see Sect. 2.2.1). However, since the overall fit is based on weak lines the column density results are not very sensitive to the exact $b$ value.

Our measurements of column densities for the fine-structure levels of the $\mathrm{Fe}^{+}$ground-term $\left({ }^{6} \mathrm{D}\right)$ are in most cases consistent with those derived by D'Elia et al. (2007) and Prochaska et al. (2007a). This comparison takes into account the fact that the oscillator strengths we have adopted differ slightly (by 10-30\%) from those compiled by Morton (2003), which were adopted in previous analyses. The only exception to this consistency is the ${ }^{6} \mathrm{D}_{1 / 2}$ level, whose $\lambda 1639$ transition line is not detected in the second-epoch UVES spectrum (see Fig. 4) while Prochaska et al. (2007a) report a column density of $\log N=13.65 \pm 0.07$. This is 0.65 dex higher than our $3 \sigma$ detection limit, $\log N<13.0$. However, the latter may be biased low due to our continuum normalisation being affected by the presence of telluric absorption features, which are conspicuous in this part of the spectrum as seen from the telluric absorption-line template (Fig. 4). The 

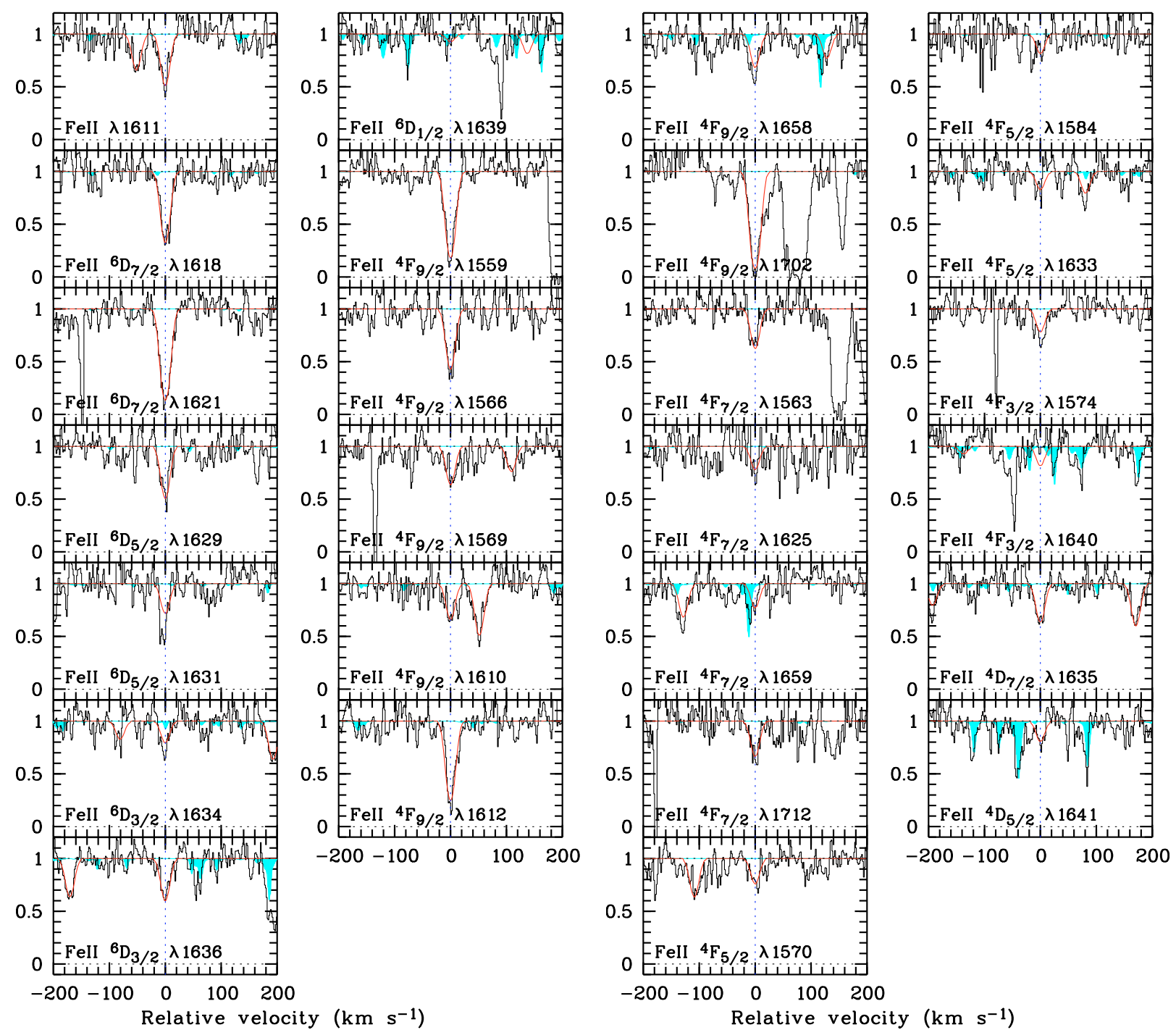

Fig. 4. Voigt-profile fitting to transition lines from the fine-structure levels of the ground term and other metastable levels of $\mathrm{Fe}^{+}$at the GRB 050730DLA redshift of $z_{\mathrm{abs}}=3.96857$ (taken as the origin of the velocity scale) in the second-epoch UVES observations. The lower level of the transitions, for which the column density is determined from the fits, is indicated in each panel, except for the lowest $\mathrm{Fe}^{+}$energy level (ground state) corresponding to ${ }^{6} \mathrm{D}_{9 / 2}$. The shaded regions in some of the panels, most notably at rest-frame wavelengths of around $1640 \AA$, indicate the expected location and shape of atmospheric features from a synthetic telluric absorption-line spectrum described in Sect. 2.2.3.

template also matches the four absorption-line features at -120 , $-80,0$, and $+80 \mathrm{~km} \mathrm{~s}^{-1}$ observed around $8145.5 \AA$ in the MIKE spectrum (see bottom right panel of Fig. 3 in Prochaska et al. 2007a), both in terms of wavelength positions and relative optical depths, pointing to significantly higher amount of precipitable water vapour present, during the observations of GRB 050730, in the atmosphere above Las Campanas observatory than on Paranal. From this, we conclude that the actual $\mathrm{Fe}^{+}{ }^{6} \mathrm{D}_{1 / 2}$ column density must be smaller than 13.65 , which we conservatively adopt as an upper limit in Table 2.

Taken altogether, the column densities measured for the fine-structure levels of the $\mathrm{Fe}^{+}$ground-term $\left({ }^{6} \mathrm{D}_{7 / 2},{ }^{6} \mathrm{D}_{5 / 2}\right.$, and $\left.{ }^{6} \mathrm{D}_{3 / 2}\right)$ are consistent with the values becoming smaller with time. However, the statistical significance of this result is at most marginal when levels are considered individually. Uncertainties are large for the first epoch of observations because, at $\lambda_{\text {rest }}<$ $1300 \AA$ A, only a few lines are available for a given energy level, i.e., either a single line or two mutually blended lines (see Fig. 3). It should thus be kept in mind that for the first epoch some of the fitted lines might be blended or simply mis-identified. In addition, these lines are weak and noisy. The ${ }^{6} \mathrm{D}_{1 / 2}$ level is undetected in the first-epoch UVES spectrum with a $3 \sigma$ columndensity upper limit of 13.60 (see Fig. 3 and Table 2). In contrast, the second-epoch UVES spectrum displays a wealth of Fe II lines from metastable energy levels. Column densities for the second epoch of observations are in most cases secured from at least two well-defined lines, up to seven in the case of the ${ }^{4} \mathrm{~F}_{9 / 2}$ level. Exceptions, for which we conservatively quote upper limits or equal values in Table 2 , are the ${ }^{4} \mathrm{~F}_{3 / 2}$ level (for which the feature observed at $\lambda_{\text {rest }} \approx 1574 \AA$ is inconsistent with the nondetection of the $\lambda 1640$ line probably because of improper continuum placement) and the ${ }^{4} \mathrm{D}_{5 / 2}$ level (whose detection through the $\lambda 1641$ line is uncertain due to the presence of adjacent telluric absorption; see Fig. 4).

\section{3. $\mathrm{Fe}^{+}$photo-excitation modelling}

Our group successfully modelled the time-variable excitation of $\mathrm{Fe}^{+}$and $\mathrm{Ni}^{+}$at the host-galaxy redshift of GRB 060418 as due to pumping by afterglow ultra-violet (UV) photons 
Table 2. Column densities of the fine-structure levels of the ground term and those of metastable levels of $\mathrm{Fe}^{+}$at the GRB 050730-DLA redshift of $z_{\mathrm{abs}}=3.96857$.

\begin{tabular}{|c|c|c|c|}
\hline Ion & Energy level & $\begin{array}{l}\text { Transition } \\
\text { lines used }(\AA)\end{array}$ & $\log N \pm \sigma_{\log N}{ }^{b}$ \\
\hline \multicolumn{4}{|c|}{ 1st epoch of observations: } \\
\hline $\mathrm{Fe}^{+}$ & g.s. ${ }^{a}\left({ }^{6} \mathrm{D}_{9 / 2}\right)$ & 1083,1112 & $15.31_{-0.05}^{+0.04}$ \\
\hline $\mathrm{Fe}^{+}$ & ${ }^{6} \mathrm{D}_{7 / 2}$ & 1266,1267 & $14.39_{-0.12}^{+0.10}$ \\
\hline $\mathrm{Fe}^{+}$ & ${ }^{6} \mathrm{D}_{5 / 2}$ & 1271 & $14.36_{-0.10}^{+0.09}$ \\
\hline $\mathrm{Fe}^{+}$ & ${ }^{6} \mathrm{D}_{3 / 2}$ & $1275.7,1275.8$ & $14.04_{-0.16}^{+0.12}$ \\
\hline $\mathrm{Fe}^{+}$ & ${ }^{6} \mathrm{D}_{1 / 2}$ & $1277.64,1277.68$ & $<13.60^{c}$ \\
\hline \multicolumn{4}{|c|}{ 2nd epoch of observations: } \\
\hline $\mathrm{Fe}^{+}$ & g.s. ${ }^{a}\left({ }^{6} \mathrm{D}_{9 / 2}\right)$ & 1611 & $15.34_{-0.06}^{+0.06}$ \\
\hline $\mathrm{Fe}^{+}$ & ${ }^{6} \mathrm{D}_{7 / 2}$ & 1618,1621 & $14.41_{-0.03}^{+0.03}$ \\
\hline $\mathrm{Fe}^{+}$ & ${ }^{6} \mathrm{D}_{5 / 2}$ & 1629,1631 & $13.96_{-0.07}^{+0.06}$ \\
\hline $\mathrm{Fe}^{+}$ & ${ }^{6} \mathrm{D}_{3 / 2}$ & 1634,1636 & $13.79_{-0.09}^{+0.09}$ \\
\hline $\mathrm{Fe}^{+}$ & ${ }^{6} \mathrm{D}_{1 / 2}$ & 1639 & $<13.65^{d}$ \\
\hline $\mathrm{Fe}^{+}$ & ${ }^{4} \mathrm{~F}_{9 / 2}$ & $\begin{array}{l}1559,1566,1569 \\
1610,1612,1658\end{array}$ & \\
\hline & & 1702 & $14.31_{-0.04}^{+0.03}$ \\
\hline $\mathrm{Fe}^{+}$ & ${ }^{4} \mathrm{~F}_{7 / 2}$ & $\begin{array}{l}1563,1625,1659 \\
1712\end{array}$ & $13.64_{-0.10}^{+0.10}$ \\
\hline $\mathrm{Fe}^{+}$ & ${ }^{4} \mathrm{~F}_{5 / 2}$ & $1570,1584,1633$ & $13.50_{-0.14}^{+0.11}$ \\
\hline $\mathrm{Fe}^{+}$ & ${ }^{4} \mathrm{~F}_{3 / 2}$ & 1574,1640 & $\leq 13.39_{-0.12}^{+0.11 d}$ \\
\hline $\mathrm{Fe}^{+}$ & ${ }^{4} \mathrm{D}_{7 / 2}$ & 1635 & $13.46_{-0.08}^{+0.08}$ \\
\hline $\mathrm{Fe}^{+}$ & ${ }^{4} \mathrm{D}_{5 / 2}$ & 1641 & $\leq 13.29_{-0.15}^{+0.13 d}$ \\
\hline
\end{tabular}

${ }^{a}$ Ground state. ${ }^{b}$ Listed uncertainties were derived from the line fitting using the best-fit continuum normalisation plus or minus 0.5 times the noise rms in the adjacent continuum. ${ }^{c} 3 \sigma$ upper limit. ${ }^{d}$ Possible blend or mis-identification (see text).

(Vreeswijk et al. 2007). This led to the first determination of the distance of the bulk of the host-galaxy absorbing neutral material to a GRB $(d=1.7 \pm 0.2 \mathrm{kpc})$. The detection of absorptionline variability with a steadily rising population of $\mathrm{Fe}^{+}$in the ${ }^{4} \mathrm{~F}_{9 / 2}$ energy level, and the simultaneous decay of the other levels, ruled out collisions with electrons as the dominant source of excitation. There is now mounting evidence that excitation by UV photons and subsequent de-excitation cascades are the mechanisms at play to populate the $\mathrm{Fe}^{+}$metastable energy levels in GRB afterglow spectra, as variations of Fe II absorption lines have been observed at the host-galaxy redshifts of two additional bursts, GRB 020813 and GRB 080319B, for which suitable multi-epoch data are available (Dessauges-Zavadsky et al. 2006; D'Elia et al. 2009). In the case of GRB 050730, absorption-line variability of transitions involving the $\mathrm{Fe}^{+6} \mathrm{D}_{9 / 2}$, ${ }^{6} \mathrm{D}_{7 / 2},{ }^{6} \mathrm{D}_{5 / 2}$, and ${ }^{6} \mathrm{D}_{3 / 2}$ levels cannot be firmly established (see Sect. 2.2.3) as the difference between the two epochs of observations is small relative to the time elapsed since the burst and, therefore, collisions cannot be excluded as a possible source of excitation. Single-epoch Boltzmann fits to both the observed ground-state and the metastable level populations of $\mathrm{Fe}^{+}$, including ${ }^{4} \mathrm{~F}_{9 / 2}$ and higher energy levels, give poor results, with $\chi_{v}^{2}=13.2 /(4-2)=6.6, T_{\mathrm{ex}} \sim 400 \mathrm{~K}$, and $\log N\left(\mathrm{Fe}^{+}\right)=15.3$ for the first epoch, and $\chi_{v}^{2}=106.8 /(8-2)=17.8, T_{\mathrm{ex}} \sim 2600 \mathrm{~K}$, and $\log N\left(\mathrm{Fe}^{+}\right)=14.5$ for the second epoch (while $\log N\left(\mathrm{Fe}^{+}\right)=$ $15.34 \pm 0.06$ is observed). The very poor Boltzmann-distribution fits, as well as the disparate excitation temperatures and groundstate column densities inferred from the two quasi-simultaneous epoch data, lead us to reject the hypothesis that collisions are the cause of the excitation of $\mathrm{Fe}^{+}$. As a consequence, we apply below our UV pumping model to the case of GRB 050730.

\subsubsection{Model}

In our model, GRB afterglow photons progressively excite $\mathrm{Fe}^{+}$ions in a gas cloud located at a distance $d$ from the burst. Although we take into account both direct excitation by afterglow IR photons and indirect excitation by UV photons, the latter is, at a given distance, by far the dominant excitation mechanism (Prochaska et al. 2006; Vreeswijk et al. 2007). In addition, as a result of the large separation of the $\mathrm{Fe}^{+}$ground-term finestructure energy levels, the cosmic microwave background radiation is always a negligible source of excitation of $\mathrm{Fe}^{+}$even at $z=4$ (Silva \& Viegas 2002). The ion level populations at a given time are a function of the strength and spectral slope of the afterglow UV radiation and its decay with time, the pre-burst $\mathrm{Fe}^{+}$column density (all ions are assumed to be initially in the ground state), the distance of the absorbing cloud to the GRB explosion site, and the velocity broadening of the ions. In this respect, our analysis closely follows that of Vreeswijk et al. (2007) who modelled the case of GRB 060418. Although the narrow time window covered by the UVES observations of GRB 050730 does not allow for a definite detection of absorption-line variability (see above), unlike the UVES observations of GRB 060418, the large number of $\mathrm{Fe}^{+}$metastable energy levels detected in absorption towards GRB 050730 constrains the model quite well, as shown below, so that the burst/DLA distance can be reliably determined.

The intensity of the UV flux illuminating the cloud is obtained by converting the observed afterglow light curve to the GRB rest frame. We used the light-curve description of Pandey et al. (2006), namely a broken power law with a break time around 0.1 day with pre- and post-break power-law indices of $\alpha_{1}=-0.60 \pm 0.07$ and $\alpha_{2}=-1.71 \pm 0.06$, respectively. These authors also measured a spectral slope of $\beta=-0.56 \pm 0.06$, which we adopted in our model. Kann et al. (2007) measured similar values for these indices. For the flux zero-point of the light curve, we adopted $I=17.22$ at $t_{\mathrm{obs}}=3.47 \mathrm{~h}$ after the burst (Pandey et al. 2006); this epoch was also assumed to be the time of the jet break. We note that the $I$-band filter does not contain the $\operatorname{Ly} \alpha$ forest of absorption lines which in turn affects the $B, V$, and $R$-band photometry (see Pandey et al. 2006), while the restframe central wavelength of the $I$-band filter is around $1600 \AA$, which is in the wavelength regime (912-2600 $⿱$ ) of the UV photons responsible for the excitation of $\mathrm{Fe}^{+}$. This leads to the following description of the rest-frame flux at a distance $d$ from GRB 050730:

$$
\begin{aligned}
F_{v}^{\mathrm{rest}}= & \frac{3.21 \times 10^{-27}}{1+z} \\
& \times\left[\frac{t_{\mathrm{obs}}}{3.47 \mathrm{~h}}\right]^{\alpha}\left[\frac{\lambda_{\mathrm{obs}}}{7977 \AA}\right]^{-\beta}\left[\frac{3.55 \times 10^{10} \mathrm{pc}}{d}\right]^{2}
\end{aligned}
$$

in erg s $\mathrm{s}^{-1} \mathrm{~cm}^{-2} \mathrm{~Hz}^{-1}$. In the conversion to the rest frame, we adopted the Galactic extinction of $E(B-V)=0.046$ from Schlegel et al. (1998) and assumed that any other extinction along the line of sight is negligible (see also Starling et al. 2005). The latter assumption is consistent with the low metallicity and low depletion factor of $[\mathrm{S} / \mathrm{Fe}]$ measured at the hostgalaxy absorber redshift, which indicates a low dust content if any (see Sect. 2.2.1). For the calculation of the luminosity distance, $d_{1}=3.55 \times 10^{10} \mathrm{pc}$, we adopted $H_{0}=70 \mathrm{~km} \mathrm{~s}^{-1} \mathrm{Mpc}^{-1}$, $\Omega_{\mathrm{M}}=0.3$, and $\Omega_{\Lambda}=0.7$. For the calculation of the atom level populations, we refer the reader to Eqs. (3)-(5) and the accompanying explanations of Vreeswijk et al. (2007). We checked for the contribution of the source function by performing two runs 
including and excluding it; the change in the resulting chi-square was found to be negligible. As including the source function requires much more CPU time, we set the source function to zero. For the velocity broadening of the ions, we adopted the value that we measured in the UVES spectra, $b=10 \mathrm{~km} \mathrm{~s}^{-1}$ (see Table 2) as it is well-constrained due to the simplicity of the absorption-line profile of this GRB-DLA. For many UV transitions, the cloud that we model is optically thick and, therefore, as in Vreeswijk et al. (2007), we sliced up the cloud in a sufficient number of plane-parallel layers, so that each layer can be considered optically thin for a given transition. In contrast to GRB 060418, we fixed the starting time $t_{0}$ to $30 \mathrm{~s}$ in the rest frame for GRB 050730 as the results are very insensitive to any value of $t_{0}$ between five and $200 \mathrm{~s}$. In other words, the level populations at roughly an hour (in the rest frame) after the burst do not depend on the brightness of the very early afterglow, up to a rest-frame time of about five minutes.

\subsubsection{Atomic data}

We considered two different sets of $\mathrm{Fe}^{+}$atomic parametres in our calculations: the first one is the same set as used in Vreeswijk et al. (2007), and the second one is the atom model employed in the Cloudy photo-ionization code (see Ferland 2003) described in Verner et al. (1999). The former set of atomic data, which we refer to below as the "old atomic parametres", includes the 20 lower energy levels of $\mathrm{Fe}^{+}$(up to $E=18886.78 \mathrm{~cm}^{-1}$ ). The probabilities for spontaneous decay, or $A$ values, of the forbidden transitions between all these lower levels were taken from Quinet et al. (1996). For the allowed transitions between lower and higher (starting from $E=38458.99 \mathrm{~cm}^{-1}$ ) excited levels, we adopted the $A$ values compiled by Morton (2003) whenever available and, if not, we used those provided by Kurucz $(2003)^{5}$. The Cloudy atomic data set includes all 63 lower energy levels of $\mathrm{Fe}^{+}$. We here directly cite Verner et al. (1999) for the description of the corresponding sources: "transition probabilities are taken from theoretical calculations by Nahar (1995, allowed transitions) and Quinet et al. (1996, forbidden transitions) and supplemented by data from compilations by Fuhr et al. (1988) and Giridhar \& Arellano Ferro (1995). Transition probabilities for all intercombination lines not covered by these compilations are taken from the Kurucz \& Bell (1995) database. Uncertainties are generally smaller than $20 \%$ for strong permitted lines but can be larger than $50 \%$ for weak permitted and intercombination lines. For the forbidden transitions of interest, uncertainties are expected to be less than 50\%". Using these two different atomic data sets gives us insight into the uncertainties in the output fit parametres, most importantly the distance of the photo-excited cloud to the GRB. The final resulting uncertainties of the model parametres only reflect the uncertainties in the column density measurements and atomic data used, and do not include the fact that our model of a plane-parallel series of absorption slabs is a simplification of the true cloud structure in the host-galaxy ISM. We also note that the different spontaneous decay coefficients $A_{\mathrm{ul}}$ (or equivalently $f$ ) of each data set result in slightly different column densities measured in the spectra.

\subsubsection{Results}

We first ran our photo-excitation code using the old atomic parametres, which results in a poor fit of the data with a reduced chi-square of $\chi_{v}^{2}=236.8 /(13-3)=23.7$ (see bottom left panel

5 http://kurucz.harvard.edu of Fig. 5). The corresponding best-fit values for the distance of the cloud to the GRB explosion site and the total $\mathrm{Fe}^{+}$column density are $d=550 \pm 40 \mathrm{pc}$ and $\log N\left(\mathrm{Fe}^{+}\right)=15.25 \pm 0.02$, respectively. When employing the Cloudy atomic parametres, the fit greatly improves (see bottom middle panel of Fig. 5), with $\chi_{v}^{2}=24.8 /(13-3)=2.5$, and best-fit values of $d=470 \pm 30 \mathrm{pc}$ and $\log N\left(\mathrm{Fe}^{+}\right)=15.52 \pm 0.03$. The $1 \sigma$ errors reported here correspond to the parametre value for which the overall chi-square increases by one with respect to the minimum. This parametre value is searched for by performing several new fits where the relevant parametre is fixed, while the other parametres are left free to vary, until a fit is found where $\Delta \chi^{2}$ equals one.

The difference between the best fits, obtained using either the old atomic parametres or the Cloudy atomic parametres, mainly comes from the very different values of the spontaneous decay coefficients of the $\mathrm{Fe}^{+}{ }^{4} \mathrm{~F}_{9 / 2}$ and ${ }^{4} \mathrm{~F}_{7 / 2}$ energy levels. The lower $A$-values in the old atomic parametre set, by about a factor of three, cause these levels to be severely overestimated in the model, as evident from the bottom left panel of Fig. 5. This in turn results in the populations of the ground state and associated fine-structure levels of $\mathrm{Fe}^{+}$being underestimated. Instead, the Cloudy atomic parametre set results in a very reasonable fit as shown in the bottom middle panel of Fig. 5. However, we note that despite the large difference in the atomic data parametre sets used (both in the number of levels and $A$-values), the derived distances: $d=550 \pm 40$ pc (old set) and $d=470 \pm 30$ pc (Cloudy set) are consistent at the $1.6 \sigma$ level.

In contrast to the case of GRB 050730, the observations of GRB 060418 were successfully fit using the old atomic parametres (see Vreeswijk et al. 2007). Therefore, we verified whether the time-variability of the excited $\mathrm{Fe}^{+}$and $\mathrm{Ni}^{+}$energy-level populations observed along the line of sight to GRB 060418 can also be fit using the Cloudy atomic parametres. We find that the latter actually provides a much better fit, with a reduced chi-square $\left(\chi_{v}^{2}=19.3 /(36-5)=0.62\right)$ almost half of that of the fit using the old atomic parametres. The Cloudy best-fit distance of the absorbing cloud to the GRB $(d=2.0 \pm 0.3 \mathrm{kpc})$ is fully consistent within the errors with the previously derived value $(d=1.7 \pm 0.2 \mathrm{kpc})$, while the best-fit total $\mathrm{Fe}^{+}$column density is significantly higher: $\log N\left(\mathrm{Fe}^{+}\right)=15.36 \pm 0.11$ (to be compared to $\log N\left(\mathrm{Fe}^{+}\right)=14.75_{-0.04}^{+0.06}$ from the old fit). This updated $\mathrm{Fe}^{+}$column density is consistent with that observed along the line of sight to GRB 060418, thereby eliminating the need for another absorbing cloud, located at much larger distance from the burst, that is not significantly excited (see the discussion in Vreeswijk et al. 2007). This major improvement of the photoexcitation modelling of the GRB 060418 data lends strong additional support for adopting the Cloudy atomic parametres in our code rather than the old atomic parametres.

Up to this point, we have assumed that all the absorbing neutral material is located at the same distance along the line of sight to the GRB, i.e., that the cloud is infinitely small. In an improved version of our code, we allow the absorbing cloud to have a physical extent over which the cloud is assumed to have constant density, and consider its linear size $l$ as an additional fit parametre. Applying this more realistic model to the data, with each cloud layer (see above) now located at a different distance from the burst, leads to the following results: a burst/cloud distance (defined to the near-side of the cloud) of $d=440 \pm 30 \mathrm{pc}$, a linear cloud size (i.e., along the line of sight) of $l=520_{-190}^{+240} \mathrm{pc}$, and a total $\mathrm{Fe}^{+}$column density of $\log N\left(\mathrm{Fe}^{+}\right)=15.49 \pm 0.03$. It turns out that the goodness-of-fit improves significantly when introducing the cloud size, with $\chi_{v}^{2}=12.2 /(13-4)=1.4$ (compared to $\chi_{v}^{2}=2.5$ before), and the best-fit parametre values are 

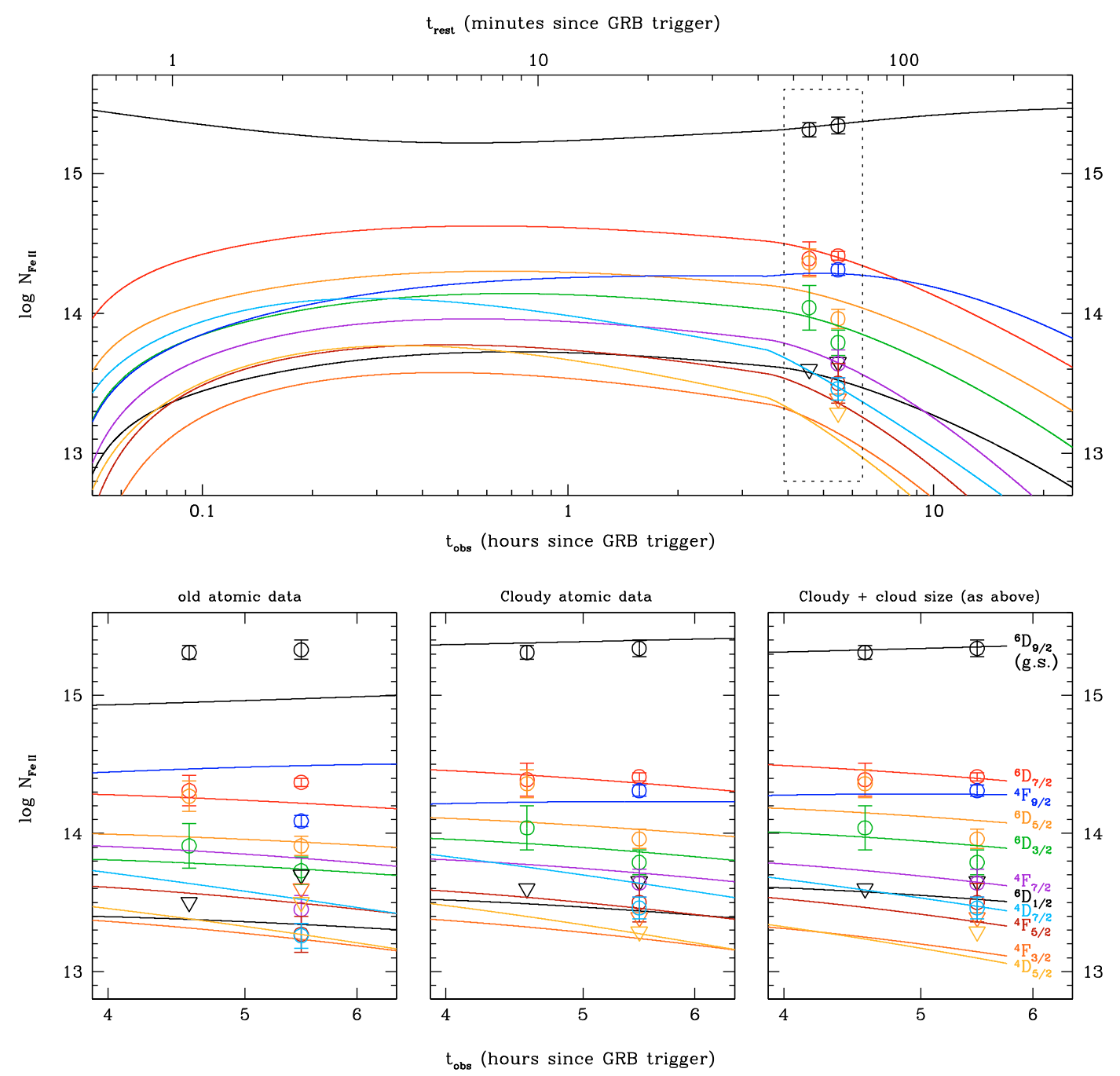

Fig. 5. Top panel: time-dependent evolution of the column densities of the ground and several metastable levels of $\mathrm{Fe}^{+}$in our best-fit $\mathrm{UV}$ pumping model (indicated with solid lines) using Cloudy atomic parametres and allowing for the absorbing cloud to be extended (see discussion in text). Measurements (resp. upper limits) are indicated with open circles (resp. triangles); the upper limits were not used to constrain the fit. Bottom panels: zoom-in around the two epochs of observations for three different model fits. The bottom right panel shows the same model as the top panel but also identifies the $\mathrm{Fe}^{+}$levels of the observed and modelled populations. The other two bottom panels show fits to the data assuming a zero cloud size, where the old atomic parametre fit is featured in the left panel and the Cloudy parametre fit is located in the middle.

well constrained. We will therefore adopt these model fit results that include a cloud size in the rest of the analysis. Although in reality the structure of ISM clouds could be much more complex than our single homogeneous cloud model, the simple singlecomponent absorption-line profile in the case of GRB 050730 (see Figs. 1, 3, and 4) indicates that our model may actually be a reasonable approximation for this burst. The fit including a cloud size is shown in both the top panel and the bottom right panel of Fig. 5. We also note that the change in light-curve decay index around the break time of $3.47 \mathrm{~h}$ is evident in the evolution of the modelled $\mathrm{Fe}^{+}$level populations.

\section{Molecular hydrogen in GRB-DLAs}

\subsection{GRB-DLA gas distance and $\mathrm{H}_{2}$ photo-dissociation}

In the previous section, we determined the second precise distance (but see also D'Elia et al. 2009) from a GRB to the bulk of the absorbing neutral material experiencing photo-excitation of its $\mathrm{Fe}^{+}$component by the time-variable GRB afterglow radiation. We found that the GRB 050730-DLA is located $0.5 \mathrm{kpc}$ away from the GRB explosion site. This distance is large enough so that the gas is not strongly ionized by the incident radiation and, therefore, remains essentially neutral, in agreement with the observations (see below). This is yet closer than what we previously determined towards GRB 060418 at $z=1.49$, where the burst/DLA distance was found to be $2 \mathrm{kpc}$ (see Sect. 2.3 and Vreeswijk et al. 2007). More recently, D'Elia et al. (2009) determined distances of 2-6 kpc for the different absorption systems in the host galaxy of GRB 080319B, using the same type of modelling as we presented in Vreeswijk et al. (2007) but with different atomic data parametres. Although it is an open question why the bulk of the absorbing neutral material is located at such distances from the GRB explosion sites, these results could be a consequence of the host galaxies of high-redshift long-duration GRBs being compact, possibly $\mathrm{H}^{0}$-rich, dwarf galaxies. This would be consistent with the low metallicity of the GRB 050730DLA which we measured to be $[\mathrm{S} / \mathrm{H}]=-2.2$ (see Sect. 2.2.1). This would also be in line with the findings of imaging and spectroscopic studies that the typical long-duration GRB host at intermediate redshift is a blue, sub-luminous, low-mass, starforming galaxy (Le Floc'h et al. 2003; Christensen et al. 2004; 

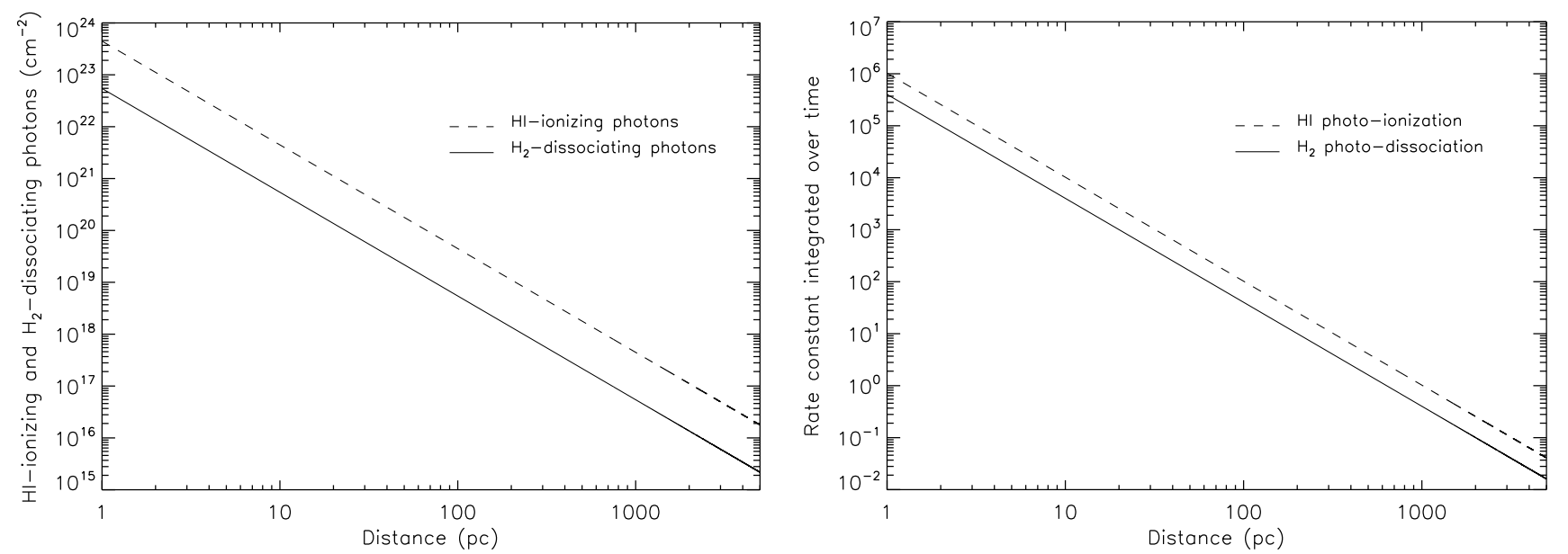

Fig. 6. Left panel: column densities of $\mathrm{H}^{0}$-ionizing and $\mathrm{H}_{2}$-dissociating photons released by GRB 050730 between 30 and $3310 \mathrm{~s}$ after the burst (rest frame; i.e., up to the mid-exposure time of the first-epoch UVES spectrum) as a function of distance to the burst. Right panel: $\mathrm{H}^{0}$ photo-ionization and $\mathrm{H}_{2}$ photo-dissociation rates integrated over the rest-frame time interval 30-3310 s as a function of distance to GRB 050730, neglecting selfshielding and assuming no dust (see text).

Chen et al. 2009; Savaglio et al. 2009). In the following, we assess in more detail the influence of the GRB afterglow radiation on the neutral $\left(\mathrm{H}^{0}\right)$ and molecular $\left(\mathrm{H}_{2}\right)$ contents of a DLA.

Using the observational constraints on the light curve described in Sect. 2.3, we can calculate the total number of $\mathrm{H}^{0}$-ionizing photons in units of $\mathrm{s}^{-1} \mathrm{~cm}^{-2}$ at a given distance from the burst as:

$n_{\text {phot }}=\frac{\int_{v_{0}}^{\infty} F_{\nu} \sigma_{\nu} \mathrm{d} v /(h v)}{\int_{v_{0}}^{\infty} \sigma_{\nu} \mathrm{d} v / v}$

where $F_{v}$ is the rest-frame GRB afterglow flux given in Eq. (1), $v_{0}$ the frequency of the hydrogen ionisation threshold $(13.6 \mathrm{eV})$, and $\sigma_{v}$ the $\mathrm{H}^{0}$ photo-ionisation cross-section. For the latter, we adopt the fits presented in Verner et al. (1996). $n_{\text {phot }}$ can be integrated over time from the observed light curve, providing the total column density of $\mathrm{H}^{0}$-ionizing photons at a distance $d$ released by the GRB. The left panel of Fig. 6 (dashed line) shows the result of this calculation for GRB 050730 photons emitted between 30 and $3310 \mathrm{~s}$ after the burst (rest frame), i.e., up to the time the first spectrum was taken. In the right panel of Fig. 6 (dashed line), we show the $\mathrm{H}^{0}$ photo-ionization rate, or $R_{\text {ion }}$. It is calculated in the same way as above except that only the numerator of Eq. (2), and not its full expression, is considered: $R_{\text {ion }}=\int_{v_{0}}^{\infty} F_{v} \sigma_{v} \mathrm{~d} v /(h v)$.

In addition, we calculate the total number of $\mathrm{H}_{2}$-dissociating photons, i.e., the number of photons in the energy range 12.24-13.51 eV (corresponding to the Lyman-Werner absorption bands), that are released by GRB 050730 between 30 and $3310 \mathrm{~s}$ after the burst (rest frame). The result is shown as a function of distance to the burst in the left panel of Fig. 6 (solid line). Only $10-15 \%$ of these photons will actually lead to dissociation of $\mathrm{H}_{2}$ molecules (Draine \& Bertoldi 1996; Hollenbach \& Tielens 1999). The $\mathrm{H}_{2}$ photo-dissociation rate is given by (see, e.g., Abel et al. 1997):

$R_{\text {diss }} \approx 1.1 \times 10^{8} J_{\mathrm{LW}} S_{\text {shield }} \mathrm{s}^{-1}$

where $J_{\mathrm{LW}}$ (in units of $\mathrm{erg} \mathrm{s}^{-1} \mathrm{~cm}^{-2} \mathrm{~Hz}^{-1}$ ) is the rest-frame GRB afterglow flux at mean energy $h \bar{v}=12.87 \mathrm{eV}$, and $S_{\text {shield }}$ a correction factor for $\mathrm{H}_{2}$ self-shielding and destruction of UV photons by dust grains (Draine \& Bertoldi 1996; see also Hirashita $\&$ Ferrara 2005). In the right panel of Fig. 6 (solid line), we plot $R_{\text {diss }}$, or equivalently $k_{27}$ (Abel et al. 1997), integrated over the rest-frame time interval $30-3310 \mathrm{~s}$ as a function of distance to the burst. We neglect self-shielding and assume no dust as the most conservative approach to reveal the maximum influence of the propagating GRB afterglow radiation. It can be seen from this figure that $R_{\mathrm{diss}}=1$ at $d \approx 500 \mathrm{pc}$ and, therefore, that $\mathrm{H}_{2}$ photo-dissociation can only be effective at $d<500$ pc.

From the definition of the rate constant, i.e., $\mathrm{d} N=R N \mathrm{~d} t$, we now calculate the $\mathrm{H}^{0}$ and $\mathrm{H}_{2}$ column densities remaining at the time the first GRB 050730 UVES spectrum was taken, i.e., $3310 \mathrm{~s}$ after the onset of GRB 050730 (rest frame), for clouds located at given distances to the burst and having different initial (pre-burst) column densities (see Fig. 7). Each calculation starts out by placing a cloud with an assumed initial column density at large distance, where $\mathrm{H}^{0}$ ionization and $\mathrm{H}_{2}$ dissociation are negligible. As the distance decreases, the cloud is increasingly affected by the afterglow photons, until the distance is reached where the entire column density is ionized or dissociated. This is shown in Fig. 7 for both $\mathrm{H}^{0}$ ionization (left panel) and $\mathrm{H}_{2}$ dissociation (right panel). The two different line types depicted in Fig. 7 correspond to two different assumptions for the cloud size: one assumes that the cloud size is negligible (solid lines), while the other assumes that the initial cloud size is equal to the burst/cloud distance. The calculations take into account the fact that the column density of photo-ionized (or photo-dissociated) particles never exceeds the column density of photons available at a given distance. We here again conservatively neglect selfshielding and, in addition, assume no dust is present, which is relevant to the case of GRB 050730.

One can see in Fig. 7 that, at typical distances of $0.5 \mathrm{kpc}$ and larger, incident GRB afterglow radiation cannot significantly ionize $\mathrm{H}^{0}$ (left panel) nor dissociate $\mathrm{H}_{2}$ (right panel) within a DLA. For a DLA cloud, i.e., with $\log N\left(\mathrm{H}^{0}\right)>20.3$, significant ionization effects can only occur at distances smaller than $50 \mathrm{pc}$. Moreover, at distances larger than $0.5 \mathrm{kpc}$ the decrease in $\mathrm{H}_{2}$ column density is smaller than 0.4 dex. Including the process of destruction of UV photons by dust grains would decrease the influence of the GRB even more. However, this will be important only for high $N\left(\mathrm{H}^{0}\right)$ values coupled to high metallicities and large dust contents, while for GRB 050730 a low dust content was inferred (see Starling et al. 2005). Therefore, the current lack of $\mathrm{H}_{2}$ detections in GRB-DLAs located at least $0.5 \mathrm{kpc}$ away 

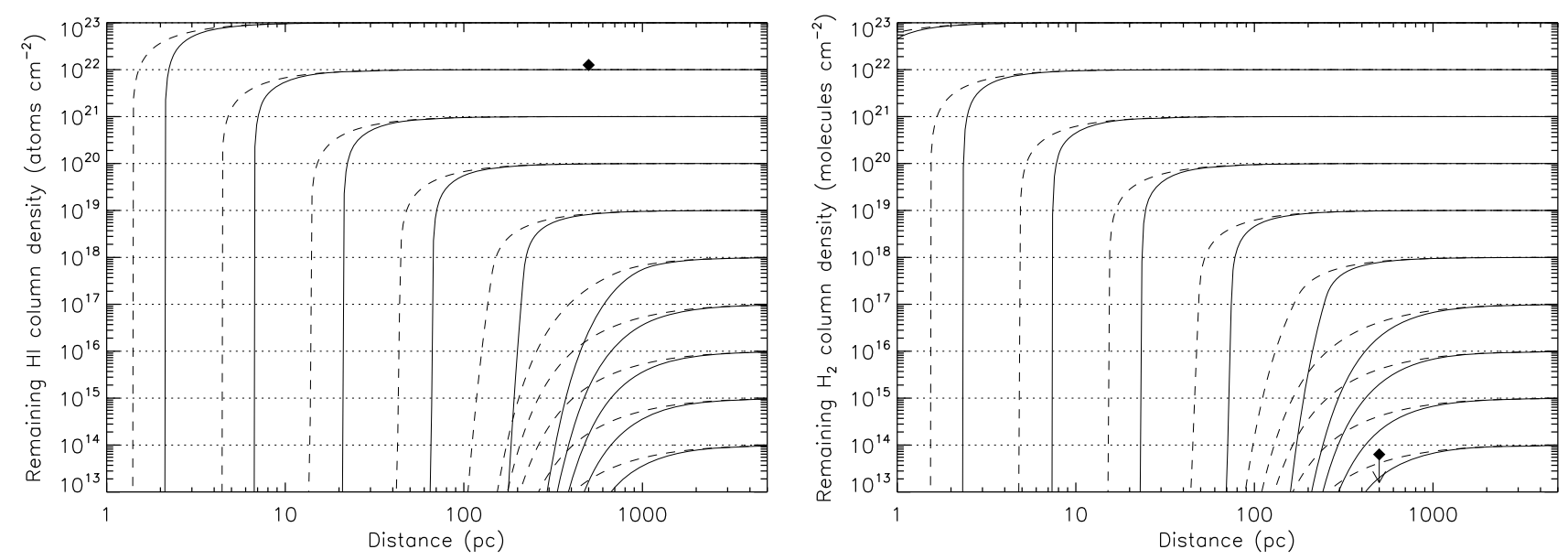

Fig. 7. Neutral hydrogen $\left(\mathrm{H}^{0}\right.$; left panel $)$ and molecular hydrogen $\left(\mathrm{H}_{2}\right.$; right panel $)$ column density remaining at the time of the first-epoch UVES spectrum as a function of distance to GRB 050730 for dust-free clouds with different initial (pre-burst) column densities. The initial column density $\left(10^{14}-10^{23}\right)$ of each calculation is indicated with a dotted horizontal line. We performed two sets of calculations: one where the cloud size is negligible (solid lines), and one where the initial cloud size is assumed to be equal to the burst/cloud distance (dashed lines). In each panel, the diamond symbol indicates our observational constraint, i.e., $\log N\left(\mathrm{H}^{0}\right)=22.10$ on the left and $\log N\left(\mathrm{H}_{2}\right)<13.8$ on the right, at a distance of $d \approx 0.5 \mathrm{kpc}$ from GRB 050730 as determined from the UV pumping model.

Table 3. Neutral gas, metals, dust, and molecules in the VLT/UVES sample of Swift-era $z_{\text {abs }}>1.8$ GRB hosts (as of June 2008).

\begin{tabular}{|c|c|c|c|c|c|c|c|c|c|}
\hline GRB & Zabs & $\log N\left(\mathrm{H}^{0}\right)$ & {$[\mathrm{X} / \mathrm{H}]$} & $\mathrm{X}$ & {$[\mathrm{X} / \mathrm{Fe}]^{c}$} & $\log N(\mathrm{Fe})_{\text {dust }}$ & $\log N\left(\mathrm{H}_{2}\right)^{f}$ & $\log f^{h}$ & $\log N\left(\mathrm{C}^{0}\right)$ \\
\hline 050730 & 3.969 & $22.10 \pm 0.10$ & $-2.18 \pm 0.11$ & $\mathrm{~S}$ & $-0.06 \pm 0.06^{d}$ & $<14.77^{d}$ & $<13.8^{g}$ & $<-8.0$ & $<13.05^{g}$ \\
\hline 050820 & 2.615 & $21.05 \pm 0.10$ & $-0.39 \pm 0.10$ & $\mathrm{Zn}$ & $+0.80 \pm 0.03$ & 16.05 & $<14.1^{g}$ & $<-6.7$ & $<12.48^{g}$ \\
\hline 050922C & 2.200 & $21.55 \pm 0.10$ & $-1.82 \pm 0.11$ & S & $+0.76 \pm 0.05^{e}$ & $<15.12^{e}$ & $<14.6^{g}$ & $<-6.7$ & $<12.45^{g}$ \\
\hline 060607 & 3.075 & $16.95 \pm 0.03$ & $\ldots^{a}$ & $\ldots^{a}$ & $\ldots^{a}$ &...$^{a}$ & $<13.5^{g}$ & $<-3.1$ & $<12.03^{g}$ \\
\hline 071031 & 2.692 & $22.15 \pm 0.05$ & $-1.73 \pm 0.05$ & $\mathrm{Zn}$ & $+0.04 \pm 0.02$ & 14.83 & $<14.1^{g}$ & $<-7.8$ & $<12.80^{g}$ \\
\hline 080310 & 2.427 & $18.70 \pm 0.10$ & $\leq-1.91 \pm 0.13^{b}$ & $\mathrm{O}^{b}$ & $\ldots^{a}$ & $\ldots^{a}$ & $<14.3^{g}$ & $<-4.2$ & $<12.55^{g}$ \\
\hline 080413A & 2.435 & $21.85 \pm 0.15$ & $-1.60 \pm 0.16$ & $\mathrm{Zn}$ & $+0.13 \pm 0.07$ & 15.13 & $<15.8^{g}$ & $<-5.7$ & $<13.28^{g}$ \\
\hline
\end{tabular}

References to the observations: 050730: D’Elia et al. (2007); 050820: Ledoux et al. (2005); 050922C: D'Elia et al. (2005); 060607: Ledoux et al. (2006); 071031: Ledoux et al. (2007); 080310: Vreeswijk et al. (2008a); 080413A: Vreeswijk et al. (2008b).

${ }^{a}$ The metallicity and/or depletion factor cannot be determined due to ionization effects.

${ }^{b}$ The OI $\lambda 1302$ line used for the fit is partially blended with strong Al II $\lambda 1670$ absorption from an intervening system at $z_{\text {abs }}=1.671$.

${ }^{c}$ The total $\mathrm{Fe}^{+}$column density includes the contributions of energy levels above the ground state up to ${ }^{4} \mathrm{~F}_{9 / 2}$ whenever transition lines from these levels were detected.

${ }^{d}$ When $[\mathrm{X} / \mathrm{Fe}]$ is negative, $N(\mathrm{Fe})_{\text {dust }}$ cannot be calculated directly. In this case, we estimate a $3 \sigma$ upper limit on $N(\mathrm{Fe})_{\text {dust }}$ by considering the upper bound provided by the $3 \sigma$ error on $[\mathrm{X} / \mathrm{Fe}]$.

${ }^{e}$ Significant overabundance of sulphur compared to iron might be present in this system (see Sect. 3.2). The column density of Fe in dust derived from $[\mathrm{S} / \mathrm{Fe}]$ (as all measurements based on $\mathrm{S}$ or $\mathrm{O}$ ) should thus be considered strictly as an upper limit.

${ }^{f}$ Sum of the contributions of the $J=0$ and 1 rotational levels.

${ }^{g} 3 \sigma$ upper limit.

${ }^{h} f \equiv 2 N\left(\mathrm{H}_{2}\right) /\left(2 N\left(\mathrm{H}_{2}\right)+N\left(\mathrm{H}^{0}\right)\right)$.

from the GRB explosion sites must be caused by one or several factors other than the propagating afterglow radiation (see also Tumlinson et al. 2007). This is what we investigate below by considering the most up-to-date sample of high-resolution VLT/UVES spectra of high-redshift GRB afterglows.

\subsection{Statistics of $\mathrm{H}_{2}$ in GRB-DLAs}

In Table 3, we list the seven Swift-era GRB afterglows with redshifts higher than 1.8 for which UVES observations were secured as of June 2008. For each line of sight, we measured in this work total $\mathrm{H}^{0}$ column densities from Voigt-profile fitting to the Ly $\alpha$ and/or Ly $\beta$ absorption lines at the GRB host-galaxy redshifts (denoted $z_{\text {abs }}$ in the table). In the same way as described in Sect. 2.2.1, we have also measured or re-measured in an homogeneous manner, from Voigt-profile fitting to the associated metal absorption lines, metallicities, $[\mathrm{X} / \mathrm{H}] \equiv \log N(\mathrm{X}) / N(\mathrm{H})-$ $\log (\mathrm{X} / \mathrm{H})_{\odot}$, and depletion factors, $[\mathrm{X} / \mathrm{Fe}]$, where $\mathrm{X}$ is a nonrefractory reference element. $X$ is taken to be $\mathrm{Zn}$ when $\mathrm{Zn}$ II lines are detected, or $\mathrm{S}$ otherwise. For GRB hosts that do not meet the requirement for DLA absorbers $\left(\log N\left(\mathrm{H}^{0}\right)<20.3\right)$, the abundance of neutral oxygen may be used instead. This atom is linked to neutral hydrogen by a strong charge-exchange reaction due to the similarity of ionization potential (Field \& Steigman 1971), and the $\left[\mathrm{O}^{0} / \mathrm{H}^{0}\right]$ ratio can lead to an estimate of the total oxygen abundance (Viegas 1995). The ionization correction factor is smaller than 10\% for GRB 080310 having a logarithmic $\mathrm{H}^{0}$ column density of 18.7. However, for GRB 060607 with $\log N\left(\mathrm{H}^{0}\right)=16.95$ the ionization correction highly depends on the hydrogen particle density and, therefore, no robust metallicity estimate can be derived. To measure depletion factors and get a handle on the dust content of the systems, iron was selected as a proxy for refractory elements. Total $\mathrm{Fe}^{+}$column 

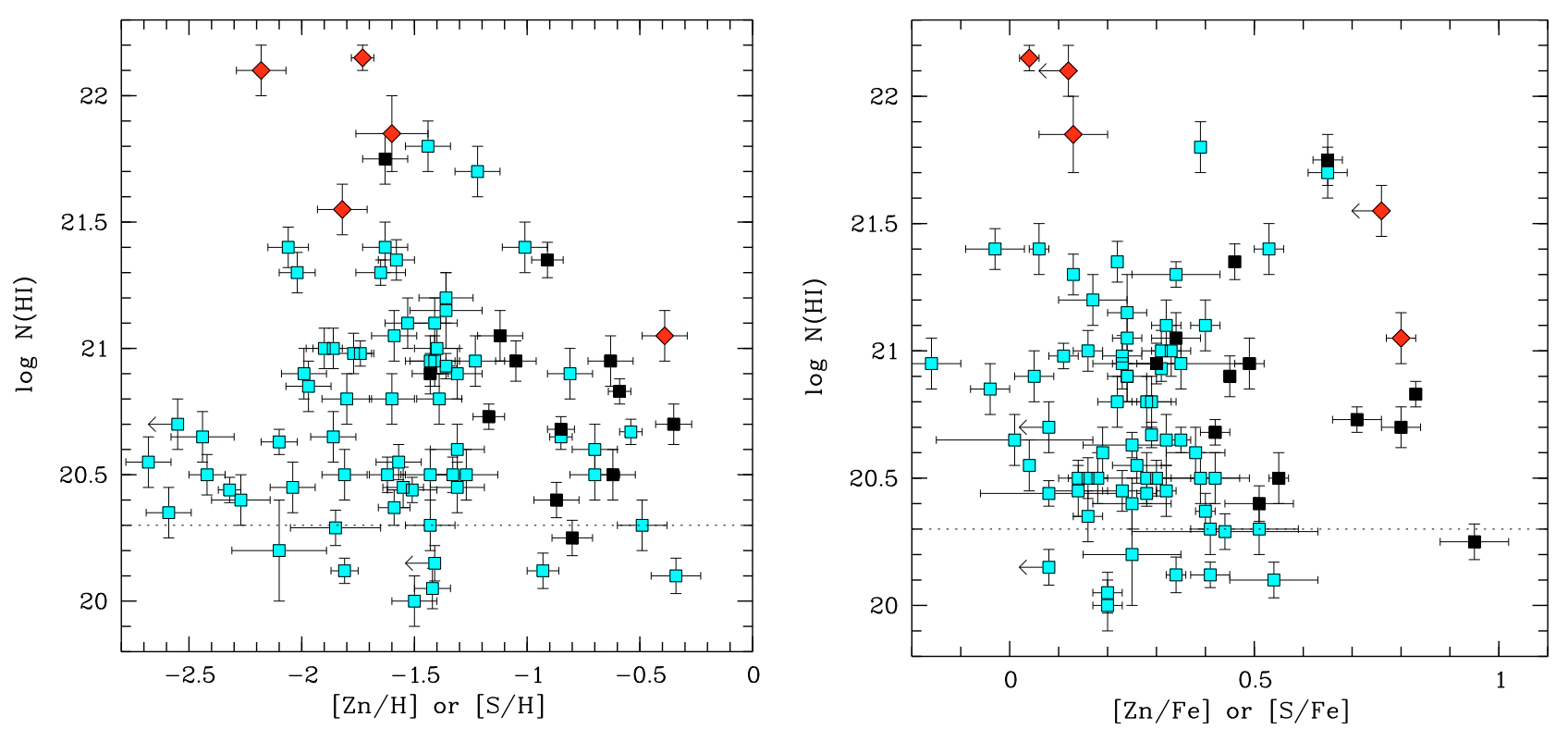

Fig. 8. Left panel: logarithm of the neutral hydrogen column density versus metallicity, $[\mathrm{X} / \mathrm{H}] \equiv \log N(\mathrm{X}) / N(\mathrm{H})-\log (\mathrm{X} / \mathrm{H})_{\odot}$, with $\mathrm{X}=\mathrm{Zn}$ or $\mathrm{S}$ (see text) in the UVES GRB-DLA absorber sample (red diamonds). Similar measurements in the sample of QSO-DLAs observed with UVES (Noterdaeme et al. 2008) are shown with squares. Black squares are for $\mathrm{H}_{2}$-bearing QSO-DLAs and blue ones for $\mathrm{H}_{2}$ non-detections. There is no $\mathrm{H}_{2}$ detection in the GRB-DLA sample. Right panel: same as before but as a function of depletion factor, [X/Fe]. Due to their very low $\mathrm{H}^{0}$ column densities, GRB 060607 and GRB 080310 are not featured in these plots.

densities were calculated by summing up the contributions of both the ground state and the metastable energy levels of $\mathrm{Fe}^{+}$ when detected. The contribution of the latter levels never exceeds 0.2 dex. In addition, we note that the corresponding absorption lines are not detected at all in the DLAs towards GRB 050820 and GRB 080413A.

$\mathrm{H}_{2}$ is not detected in any of the systems of the sample. In Table 3, we give $3 \sigma$ upper limits on the molecular hydrogen column densities and corresponding $\mathrm{H}_{2}$ molecular fractions, $f \equiv 2 N\left(\mathrm{H}_{2}\right) /\left(2 N\left(\mathrm{H}_{2}\right)+N\left(\mathrm{H}^{0}\right)\right)$. We also give $3 \sigma$ upper limits on $N\left(\mathrm{C}^{0}\right)$.

Apart from the DLA towards GRB 050820 where the metallicity is as high as $[\mathrm{Zn} / \mathrm{H}]=-0.4$, the GRB host galaxies in the UVES sample have low metallicities, $-2.2<[\mathrm{X} / \mathrm{H}]<-1.3$. In addition, the depletion factors are usually quite small, as expected for metal-poor environments having a low dust content. The only exception to this is the GRB 050922C-DLA for which sulphur is used as a proxy for non-refractory elements. In this particular case, the S II line profile does not follow that of Fe II at all, exhibiting an inversely asymmetric shape which peaks at the velocity of the detected Fe II ${ }^{\star}$ absorption. Significant overabundance of $\alpha$-elements and/or ionization effects might be present in this system and bias the observed $[\mathrm{S} / \mathrm{Fe}]$ ratio high. More generally, the $[\mathrm{S} / \mathrm{H}]$ and $[\mathrm{S} / \mathrm{Fe}]$ ratios given in Table 3 should be considered cautiously as they are strictly speaking upper limits to the true metallicities and dust depletion factors. This makes the result that both metallicities and dust depletion factors in the sample of GRB-DLAs observed with UVES (and currently all other high-resolution spectrographs) are usually modest, even stronger. Interestingly, this is most clearly seen in the three systems exhibiting the highest neutral hydrogen column densities, $\log N\left(\mathrm{H}^{0}\right) \sim 22$, where $[\mathrm{X} / \mathrm{H}]<-1.5$ and $[\mathrm{X} / \mathrm{Fe}]<+0.2$. The nature of these systems is discussed in Sect. 3.3.

The observed $\mathrm{H}^{0}$ column density distribution of Swift-era GRB-DLAs is relatively flat and not highly skewed towards extremely high values (see Jakobsson et al. 2006; and also Table 3). In addition, because there is mounting evidence that the bulk of the $\mathrm{H}^{0}$ gas in GRB-DLAs has a galactic origin, with distances from the burst larger than $100 \mathrm{pc}$ up to kiloparsecs (see Sects. 2.3 and 3.1), the comparison of GRB-DLA properties to results drawn from QSO-DLA samples must be relevant. This is corroborated by our finding that the DLA cloud in the case of GRB 050730 is smooth and diffuse, and not apparently perturbed by star-forming regions, with a broadening parametre and physical size that are typical of the Galactic ISM. In Fig. 8, we compare the $\mathrm{H}^{0}$ column densities with metallicities and dust depletion factors for the UVES GRB-DLA sample with QSODLAs. Studies of $\mathrm{H}_{2}$ in large QSO-DLA samples observed with VLT/UVES (Ledoux et al. 2003; Noterdaeme et al. 2008) have shown that the presence of $\mathrm{H}_{2}$ does not strongly depend on the total $\mathrm{H}^{0}$ column density, and that the probability of finding $\mathrm{H}_{2}$ with high molecular fractions, $\log f>-4.5$, is only marginally higher for $\log N\left(\mathrm{H}^{0}\right)>20.8$ than below this limit $(19 \%$ and $7 \%$ respectively). The probability of $\mathrm{H}_{2}$ detection is of the order of $10 \%$ for the entire QSO-DLA population, but is actually strongly dependent on the metallicity, rising up to $35 \%$ for systems with $[\mathrm{X} / \mathrm{H}] \geq-1.3$ (Petitjean et al. 2006; Noterdaeme et al. 2008). In contrast, only about $4 \%$ of the $[\mathrm{X} / \mathrm{H}]<-1.3$ systems have $\log f>-4.5$. Applying this number statistics to the UVES GRB-DLA sample (see Table 3), composed of one $[\mathrm{X} / \mathrm{H}]>-1.3$ and four $[\mathrm{X} / \mathrm{H}]<-1.3$ DLAs, leads to a binomial probability of detecting $\mathrm{H}_{2}$ at least once of $45 \%$. This is consistent with the fact that no detection was achieved in practice.

To estimate the dust content of DLAs, it is useful to calculate the column density of iron in dust, $N(\mathrm{Fe})_{\text {dust }}=$ $\left(1-10^{-[\mathrm{X} / \mathrm{Fe}]}\right) N(\mathrm{X})(\mathrm{Fe} / \mathrm{X})_{\mathrm{dla}}$ (Vladilo et al. 2006). In their survey of 77 QSO-DLAs, Noterdaeme et al. (2008) have shown that all $\mathrm{H}_{2}$-bearing systems have $\log N(\mathrm{Fe})_{\text {dust }}>14.7$. In addition, about $17 \%$ of the systems with $14.7<\log N(\mathrm{Fe})_{\text {dust }}<$ 15.2 , and $55 \%$ of the systems above this limit, have detected $\mathrm{H}_{2}$ lines with $\log f>-4.5$. The $\log N(\mathrm{Fe})_{\text {dust }}$ values pertaining to GRB-DLAs are given in Table 3. Except in the case of the high-metallicity DLA towards GRB 050820, which exhibits $\log N(\mathrm{Fe})_{\text {dust }} \sim 16$, GRB-DLA values lie either within (in three 
cases) or below (in one case) the above range. Therefore, from QSO-DLA statistics the binomial probability of not detecting $\mathrm{H}_{2}$ at all in the UVES GRB-DLA sample is $26 \%$. From a $1.1 \sigma$ result, relying on the existence of a single system, it would be premature to conclude that GRB-DLA observations are inconsistent with known QSO-DLA properties. This is in contrast with the claim by Tumlinson et al. (2007), based on an even smaller and inhomogeneous sample of three high- and two mediumresolution GRB afterglow spectra, that $\mathrm{H}_{2}$ is generally deficient in GRB-DLAs compared to QSO lines of sight.

\subsection{Gas physical conditions}

The current lack of $\mathrm{H}_{2}$ detection along the lines of sight to GRBs might be related to the physical nature of DLAs exhibiting the highest neutral hydrogen column densities, $\log N\left(\mathrm{H}^{0}\right) \sim 22$. Indeed, these systems make up about half of the UVES GRB-DLA sample (i.e., the systems towards GRB 050730, GRB 071031 and GRB 080413A). Interestingly, three systems in the large QSO-DLA sample studied by Noterdaeme et al. (2008) share similar properties with no detected $\mathrm{H}_{2}$ but some of the highest column densities of iron in dust $\left(\log N(\mathrm{Fe})_{\text {dust }}>15.5\right)$ as well as the highest neutral hydrogen column densities ever measured along QSO lines of sight (i.e., $\log N\left(\mathrm{H}^{0}\right)=21.70$ towards Q $0458-0203, \log N\left(\mathrm{H}^{0}\right)=21.80$ towards Q $1157+0128$, and $\log N\left(\mathrm{H}^{0}\right)=21.40$ towards Q 1209+0919). The much smaller incidence rate of such DLAs in QSO samples is probably due to the narrow range of galactocentric radii probed by GRB afterglow observations. The properties of these systems are at variance with Galactic lines of sight, where clouds with $\log N\left(\mathrm{H}^{0}\right)>21$ usually have $\log N\left(\mathrm{H}_{2}\right)>19$ (see, e.g., Savage et al. 1977; Jenkins \& Shaya 1979).

Tumlinson et al. (2007) argue that UV radiation fields from recently formed hot stars with intensities 10-100 times the Galactic mean value must be invoked to explain GRB-DLA observations. This is conceivable if the clouds are embedded in or are located close to regions of intense star formation (see also Chen et al. 2007). However, as noted by Tumlinson et al. (2007) this contrasts with the environment of massively star-forming regions like 30 Doradus in the LMC where the existence of clouds with high $\mathrm{H}_{2}$ molecular fractions is ubiquitous (Bluhm $\&$ de Boer 2001; Danforth et al. 2002). In other words, the presence of intense UV radiation fields does not necessarily prevent $\mathrm{H}_{2}$ from forming.

In addition to a low dust content (see Sect. 3.2), and the correspondingly low formation rate of $\mathrm{H}_{2}$ onto dust grains, another reason why $\mathrm{H}_{2}$ is not detected in those QSO- and GRB-DLAs with high $\mathrm{H}^{0}$ column densities could be the particle density in the neutral gas being too small. For a cloud at equilibrium, by equating the $\mathrm{H}_{2}$ formation $\left(R_{\text {dust }}\right)$ and photo-dissociation $\left(R_{\text {diss }}\right)$ rates, one can write (see, e.g., Hirashita \& Ferrara 2005):

$R_{\text {diss }} N\left(\mathrm{H}_{2}\right)=n_{\mathrm{H}} R_{\text {dust }} N(\mathrm{H})$

where $N(\mathrm{H})$ is the total hydrogen column density and $N\left(\mathrm{H}_{2}\right)$ the molecular hydrogen column density, which then linearly depends on the particle density, $n_{\mathrm{H}}$. Therefore, the lower the density the lower the molecular fraction. In the case of the DLA towards GRB 050730, from its high $\mathrm{H}^{0}$ column density, $\log N\left(\mathrm{H}^{0}\right)=22.10$, and considering the best-fit linear cloud size of $l=520_{-190}^{+240} \mathrm{pc}$, a meaningful order of magnitude estimate of the particle density can be inferred: $n_{\mathrm{H}} \approx 5-15 \mathrm{~cm}^{-3}$. Using Eq. (4) and the prescriptions by Hirashita \& Ferrara (2005), we can estimate the UV intensity at $h v=12.87 \mathrm{eV}$ outside the
GRB 050730-DLA cloud, averaged over the solid angle, taking into account $\mathrm{H}_{2}$ self-shielding. In order to keep the amount of $\mathrm{H}_{2}$ below the observational threshold, $\log N\left(\mathrm{H}_{2}\right)<13.8$ (see Table 3), the UV intensity must be at least of the order of the Galactic radiation field: $J_{\mathrm{LW}} \gtrsim 3 \times 10^{-20} \mathrm{erg} \mathrm{s}^{-1} \mathrm{~cm}^{-2} \mathrm{~Hz}^{-1} \mathrm{sr}^{-1}$. This differs by more than a factor of ten from the results by Tumlinson et al. (see above). This is because in the latter study a scaling of the Galactic $\mathrm{H}_{2}$ formation rate based on the metallicity rather than on the dust-to-gas ratio, $\kappa=10^{[\mathrm{X} / \mathrm{H}]}\left(1-10^{[\mathrm{Fe} / \mathrm{X}]}\right)$, was assumed or, equivalently, all metals are incorporated into dust grains. However, a strong correlation between depletion factor and metallicity is observed in QSO-DLAs (see, e.g., Ledoux et al. 2003), with smaller depletion factors in low metallicity systems, indicating that the dust-to-metal ratio increases markedly in the course of chemical evolution (Vladilo 2004).

While, as shown above, particle densities are probably at most moderate in GRB-DLAs, with $n_{\mathrm{H}} \approx 5-15 \mathrm{~cm}^{-3}$, the ambient UV radiation field intensities needed to explain the lack of $\mathrm{H}_{2}$ in systems with high $\mathrm{H}^{0}$ column densities are only of the order of 1-10 times those observed in the Galaxy, thus not particularly high. As shown in Sect. 3.2, the main factor driving the absence of $\mathrm{H}_{2}$ in the current sample of GRB-DLAs observed with VLT/UVES is a combination of low metallicities and low dust contents.

\section{Summary and prospects}

In this paper, we have presented a detailed analysis of the DLA cloud $\left(\log N\left(\mathrm{H}^{0}\right)=22.10\right)$ at the GRB 050730 host-galaxy redshift of $z_{\mathrm{abs}}=3.969$ using high-quality VLT/UVES spectra. Accurate metal abundances are derived showing that this GRB-DLA has both low metallicity, $[\mathrm{S} / \mathrm{H}]=-2.18 \pm 0.11$, and no dust. In addition, we measured $[\mathrm{N} / \mathrm{S}]=-0.98 \pm 0.05$ and $[\mathrm{Ar} / \mathrm{S}]=-0.11 \pm 0.06$. The former ratio is indicative of a high nitrogen enrichment dominated by intermediate-mass stars (4-8 $M_{\odot}$ ), before significant oxygen production by supernovae, and a past low star-formation efficiency (e.g., Henry \& Prochaska 2007; Petitjean et al. 2008). The latter ratio is a tracer of the radiation field in $\mathrm{H}^{0}$ regions (Vladilo et al. 2003). The observed high abundance of argon, typical of the local ISM, is consistent with the expectation for a neutral medium embedded in a soft ionizing continuum.

From the self-consistent photo-excitation modelling of absorption lines from electronic transitions to an unprecedentedly large number of $\mathrm{Fe}^{+}$energy levels, the distance of the absorbing neutral material to GRB 050730 is constrained to be $d \approx 0.5 \mathrm{kpc}$. Similar photo-excitation modelling applied to the observed column densities and upper limits of $\mathrm{S}^{3+}$ energy levels has led to a lower limit on the distance of the $\mathrm{S}^{3+}$-bearing gas of $0.4 \mathrm{kpc}$ (Fox et al. 2008). From detailed calculations, we find that at such distances GRB afterglow photons cannot ionize $\mathrm{H}^{0}$ nor dissociate $\mathrm{H}_{2}$ significantly. From the above $\mathrm{Fe}^{+}$column density modelling, a linear cloud size of $l=520_{-190}^{+240} \mathrm{pc}$ is inferred, suggesting that GRB-DLAs typically exhibiting very high $\mathrm{H}^{0}$ column densities are diffuse metal-poor atomic clouds with large physical extents, $l \gtrsim 100 \mathrm{pc}$, and, at most, moderate particle densities, $n_{\mathrm{H}} \approx 5-15 \mathrm{~cm}^{-3}$.

In order to understand the lack of $\mathrm{H}_{2}$ further, we have built up the most up-to-date sample of Swift-era $z>1.8$ GRB-DLAs observed with VLT/UVES. We showed that the current nondetections of $\mathrm{H}_{2}$ in GRB-DLAs are consistent with QSO-DLA statistics where $\mathrm{H}_{2}$ is present with high molecular fractions, $\log f>-4.5$, in only $10 \%$ of the global QSO-DLA population. Moreover, the lack of $\mathrm{H}_{2}$ in the GRB-DLA sample can be 
entirely explained by the low metallicities, $[\mathrm{X} / \mathrm{H}]<-1$, and low dust contents of the systems. With the possible exception of the high-metallicity DLA towards GRB 050820, there is no need for enhanced UV radiation fields from recently formed hot stars to explain the lack of $\mathrm{H}_{2}$ (but see Tumlinson et al. 2007; see also Chen et al. 2009, who derived high mean UV radiation field intensities for four bright GRB host galaxies, whose associated DLAs all have fairly high metallicities). The additional non-detections of $\mathrm{C}$ I absorption lines, with $\log N\left(\mathrm{C}^{0}\right) / N\left(\mathrm{~S}^{+}\right)<$ -3 , suggest a warm neutral medium, with kinetic temperatures $T_{\text {kin }} \gtrsim 1000 \mathrm{~K}$, as found in most QSO-DLAs (Srianand et al. 2005).

The evidence for low to very low GRB-DLA metallicities coming from high spectral resolution observations is in contrast with the current sample of metallicity and depletion measurements drawn from low- and/or intermediate-resolution spectroscopy (e.g., Fynbo et al. 2006; Savaglio 2006; Prochaska et al. 2007b) which points towards higher metallicities than in the QSO-DLA population (see also Fynbo et al. 2008, who tried to reconcile the different metallicity distributions of QSOand GRB-DLAs). Moreover, hints at the presence of $\mathrm{H}_{2}$ and/or $\mathrm{C}$ I lines have been reported at the host-galaxy redshifts of GRB 050401 (Watson et al. 2006), GRB 060206 (Fynbo et al. 2006), and GRB 070802 (Elíasdóttir et al. 2009), while the metallicities of these hosts are amongst the highest measured in GRB-DLAs. This suggests that it is only a matter of time before $\mathrm{H}_{2}$ and/or other molecules are detected beyond any doubt in GRB-DLAs ${ }^{6}$. Care should be exercized, however, not to overinterpret the current inhomogeneous body of data, as claims for high $\mathrm{Zn}^{+}$column densities and depletion factors of zinc compared to iron are based on a handful of absorbers at intermediate redshifts, $1<z<2$, for which $N\left(\mathrm{H}^{0}\right)$ measurements are lacking (Savaglio 2006).

The VLT/UVES sample of high-redshift GRB afterglows shows a lack of GRB-DLAs with both high $\mathrm{H}^{0}$ column density and high metallicity (see Fig. 8, left panel). However, this does not mean that such systems do not exist and an anti-correlation between absorber dust content and afterglow optical brightness should be checked. Indeed, the associated extinction will be high in these systems (Vladilo \& Péroux 2005; see also Prochaska et al. 2009) and their observation with high-resolution spectrographs, even with the advantage of fast response, possibly difficult. This is consistent with the findings of multi-colour photometry that a large fraction of bursts are dark because of high dust extinction in their hosts (Rol et al. 2007; Jaunsen et al. 2008; Tanvir et al. 2008; Cenko et al. 2009). If a bias exists in the UVES sample, it could also be against GRB-DLAs located at much smaller distances from their bursts than currently found because, as we have shown, high metal (resp. $\mathrm{H}_{2}$ ) column densities are able to survive the ionizing (resp. dissociating) effects of the incident GRB afterglow radiation.

This potential problem should be alleviated by the secondgeneration VLT instrument X-Shooter, currently in its commissioning phase, to complement the capabilities of UVES at the VLT, Unit 2 - Kueyen, telescope. X-Shooter will be the instrument of choice to cover any redshift from $z=0.3$ up to $z=10$ for $1600 \AA$ (rest frame) Fe II and other metal lines in GRB afterglows fainter, or redder, than observable with UVES. In the

\footnotetext{
${ }^{6}$ Since the submission of this paper, CO molecules with exceptionally high column densities have been detected at the host-galaxy redshift of GRB 080607 in a low-resolution Keck afterglow spectrum (Prochaska et al. 2009). The absorbing gas is estimated to have roughly solar metallicity.
}

meantime, we plan to apply the techniques presented in this paper for GRB 050730 to other GRB lines of sight observed with UVES to start building up a sample of burst/DLA distance determinations and map the distribution of neutral gas in GRB host galaxies.

Acknowledgements. P.M.V. acknowledges the support of the EU under a Marie Curie Intra-European Fellowship, contract MEIF-CT-2006-041363. The Dark Cosmology Centre is funded by the Danish National Research Foundation.

\section{References}

Abel, T., Anninos, P., Zhang, Y., \& Norman, M. L. 1997, New Astronomy, 2, 181

Abgrall, H., Roueff, E., Launay, F., \& Roncin, J.-Y. 1994, Canadian J. Phys., 72, 856

Berger, E., Penprase, B. E., Cenko, S. B., et al. 2006, ApJ, 642, 979

Bluhm, H., \& de Boer, K. S. 2001, A\&A, 379, 82

Cenko, S. B., Kelemen, J., Harrison, F. A., et al. 2009, ApJ, 693, 1484

Chen, H.-W., Prochaska, J. X., Bloom, J. S., \& Thompson, I. B. 2005, ApJ, 634, L25

Chen, H.-W., Prochaska, J. X., \& Bloom, J. S. 2007, ApJ, 668, 384

Chen, H.-W., Perley, D. A., Pollack, L. K., et al. 2009, ApJ, 691, 152

Christensen, L., Hjorth, J., \& Gorosabel, J. 2004, A\&A, 425, 913

Danforth, C. W., Howk, J. C., Fullerton, A. W., Blair, W. P., \& Sembach, K. R. 2002, ApJS, 139, 81

Dekker, H., D’Odorico, S., Kaufer, A., Delabre, B., \& Kotzlowski, H. 2000, in Optical and IR Telescope Instrumentation and Detectors, ed. M. Iye, \& A. F. Moorwood, SPIE Conf., 4008, 534

D'Elia, V., Piranomonte, S., Fiore, F., et al. 2005, GRB Coordinates Network, 4044, 1

D’Elia, V., Fiore, F., Meurs, E. J. A., et al. 2007, A\&A, 467, 629

D'Elia, V., Fiore, F., Perna, R., et al. 2009, ApJ, 694, 332

Dessauges-Zavadsky, M., Chen, H.-W., Prochaska, J. X., Bloom, J. S., \& Barth, A. J. 2006, ApJ, 648, L89

Draine, B. T., \& Bertoldi, F. 1996, ApJ, 468, 269

Draine, B. T., \& Hao, L. 2002, ApJ, 569, 780

Elíasdóttir, Á., Fynbo, J. P. U., Hjorth, J., et al. 2009, ApJ, 697, 1725

Ferland, G. J. 2003, ARA\&A, 41, 517

Field, G. B., \& Steigman, G. 1971, ApJ, 166, 59

Fiore, F., D'Elia, V., Lazzati, D., et al. 2005, ApJ, 624, 853

Fox, A. J., Ledoux, C., Vreeswijk, P. M., Smette, A., \& Jaunsen, A. O. 2008, A\&A, 491, 189

Fuhr, J. R., Martin, G. A., \& Wiese, W. L. 1988, Atomic transition probabilities. Scandium through Manganese (New York: American Institute of Physics (AIP) and American Chemical Society)

Fynbo, J. P. U., Prochaska, J. X., Sommer-Larsen, J., Dessauges-Zavadsky, M., \& Møller, P. 2008, ApJ, 683, 321

Fynbo, J. P. U., Starling, R. L. C., Ledoux, C., et al. 2006, A\&A, 451, L47

Galama, T. J., \& Wijers, R. A. M. J. 2001, ApJ, 549, L209

Galama, T. J., Vreeswijk, P. M., van Paradijs, J., et al. 1998, Nature, 395, 670

Giridhar, S., \& Arellano Ferro, A. 1995, Rev. Mex. Astron. Astrofis., 31, 23

Henry, R. B. C., \& Prochaska, J. X. 2007, PASP, 119, 962

Hirashita, H., \& Ferrara, A. 2005, MNRAS, 356, 1529

Hjorth, J., Sollerman, J., Møller, P., et al. 2003, Nature, 423, 847

Hollenbach, D. J., \& Tielens, A. G. G. M. 1999, Rev. Mod. Phys., 71, 173

Jakobsson, P., Hjorth, J., Fynbo, J. P. U., et al. 2004, A\&A, 427, 785

Jakobsson, P., Fynbo, J. P. U., Ledoux, C., et al. 2006, A\&A, 460, L13

Jaunsen, A. O., Rol, E., Watson, D. J., et al. 2008, ApJ, 681, 453

Jenkins, E. B., \& Shaya, E. J. 1979, ApJ, 231, 55

Jenkins, E. B., \& Tripp, T. M. 2006, ApJ, 637, 548

Kann, D. A., Klose, S., Zhang, B., et al. 2007, ArXiv e-prints, 712

Kurucz, R. L. 2003, in Modelling of Stellar Atmospheres, ed. N. Piskunov, W. W. Weiss, \& D. F. Gray, IAU Symp., 210, 45

Kurucz, R. L., \& Bell, B. 1995, Atomic Line Data, ed. R. L. Kurucz, \& B. Bell, Kurucz CD-ROM No. 23 (Cambridge, Mass.: Smithsonian Astrophysical Observatory)

Le Floc'h, E., Duc, P.-A., Mirabel, I. F., et al. 2003, A\&A, 400, 499

Ledoux, C., Petitjean, P., \& Srianand, R. 2003, MNRAS, 346, 209

Ledoux, C., Vreeswijk, P., Ellison, S., et al. 2005, GRB Coordinates Network, 3860, 1

Ledoux, C., Vreeswijk, P., Smette, A., Jaunsen, A., \& Kaufer, A. 2006, GRB Coordinates Network, 5237, 1

Ledoux, C., Jakobsson, P., Jaunsen, A. O., et al. 2007, GRB Coordinates Network, 7023, 1 
Lodders, K. 2003, ApJ, 591, 1220

MacFadyen, A. I., \& Woosley, S. E. 1999, ApJ, 524, 262

Malesani, D., Tagliaferri, G., Chincarini, G., et al. 2004, ApJ, 609, L5

Morton, D. C. 1991, ApJS, 77, 119

Morton, D. C. 2003, ApJS, 149, 205

Nahar, S. N. 1995, A\&A, 293, 967

Noterdaeme, P., Ledoux, C., Petitjean, P., \& Srianand, R. 2008, A\&A, 481, 327

Pandey, S. B., Castro-Tirado, A. J., McBreen, S., et al. 2006, A\&A, 460, 415

Penprase, B. E., Berger, E., Fox, D. B., et al. 2006, ApJ, 646, 358

Petitjean, P., Ledoux, C., Noterdaeme, P., \& Srianand, R. 2006, A\&A, 456, L9

Petitjean, P., Ledoux, C., \& Srianand, R. 2008, A\&A, 480, 349

Pian, E., Mazzali, P. A., Masetti, N., et al. 2006, Nature, 442, 1011

Prochaska, J. X., Chen, H.-W., \& Bloom, J. S. 2006, ApJ, 648, 95

Prochaska, J. X., Chen, H.-W., Bloom, J. S., et al. 2007a, ApJS, 168, 231

Prochaska, J. X., Chen, H.-W., Dessauges-Zavadsky, M., \& Bloom, J. S. 2007b, ApJ, 666, 267

Prochaska, J. X., Dessauges-Zavadsky, M., Ramirez-Ruiz, E., \& Chen, H.-W. 2008, ApJ, 685, 344

Prochaska, J. X., Sheffer, Y., Perley, D. A., et al. 2009, ApJ, 691, L27

Quinet, P., Le Dourneuf, M., \& Zeippen, C. J. 1996, A\&AS, 120, 361

Reichart, D. E., \& Price, P. A. 2002, ApJ, 565, 174

Rol, E., van der Horst, A., Wiersema, K., et al. 2007, ApJ, 669, 1098

Rothman, L. S., Jacquemart, D., Barbe, A., et al. 2005, J. Quant. Spectr. Radiat. Transf., 96, 139

Savage, B. D., Bohlin, R. C., Drake, J. F., \& Budich, W. 1977, ApJ, 216, 291

Savaglio, S. 2006, New J. Phys., 8, 195

Savaglio, S., Glazebrook, K., \& LeBorgne, D. 2009, ApJ, 691, 182

Schlegel, D. J., Finkbeiner, D. P., \& Davis, M. 1998, ApJ, 500, 525

Silva, A. I., \& Viegas, S. M. 2002, MNRAS, 329, 135
Smette, A., Horst, H., \& Navarrete, J. 2008, in The 2007 ESO Instrument Calibration Workshop (Springer-Verlag), ESO Astrophys. Symp., 433

Srianand, R., Petitjean, P., Ledoux, C., Ferland, G., \& Shaw, G. 2005, MNRAS, 362,549

Stanek, K. Z., Matheson, T., Garnavich, P. M., et al. 2003, ApJ, 591, L17

Starling, R. L. C., Vreeswijk, P. M., Ellison, S. L., et al. 2005, A\&A, 442, L21

Tanvir, N. R., Levan, A. J., Rol, E., et al. 2008, MNRAS, 388, 1743

Thöne, C. C., Wiersema, K., Ledoux, C., et al. 2008, A\&A, 489, 37

Tumlinson, J., Prochaska, J. X., Chen, H.-W., Dessauges-Zavadsky, M., \& Bloom, J. S. 2007, ApJ, 668, 667

Verner, D. A., \& Ferland, G. J. 1996, ApJS, 103, 467

Verner, D. A., Ferland, G. J., Korista, K. T., \& Yakovlev, D. G. 1996, ApJ, 465, 487

Verner, E. M., Verner, D. A., Korista, K. T., et al. 1999, ApJS, 120, 101

Viegas, S. M. 1995, MNRAS, 276, 268

Vladilo, G. 2004, A\&A, 421, 479

Vladilo, G., \& Péroux, C. 2005, A\&A, 444, 461

Vladilo, G., Centurión, M., D’Odorico, V., \& Péroux, C. 2003, A\&A, 402, 487

Vladilo, G., Centurión, M., Levshakov, S. A., et al. 2006, A\&A, 454, 151

Vreeswijk, P. M., Ellison, S. L., Ledoux, C., et al. 2004, A\&A, 419, 927

Vreeswijk, P. M., Ledoux, C., Smette, A., et al. 2007, A\&A, 468, 83

Vreeswijk, P. M., Jakobsson, P., Jaunsen, A. O., Oslo, U., \& Ledoux, C. 2008a, GRB Coordinates Network, 7391, 1

Vreeswijk, P. M., Thöne, C. C., Malesani, D., et al. 2008b, GRB Coordinates Network, 7601, 1

Watson, D., Fynbo, J. P. U., Ledoux, C., et al. 2006, ApJ, 652, 1011

Whalen, D., Prochaska, J. X., Heger, A., \& Tumlinson, J. 2008, ApJ, 682, 1114

Woosley, S. E. 1993, ApJ, 405, 273

Woosley, S. E., \& Heger, A. 2004, in Stellar Rotation, ed. A. Maeder, \& P. Eenens, IAU Symp., 215, 601 